\title{
An Efficient Two-Objective Hybrid Local Search Algorithm for Solving the Fuel Consumption Vehicle Routing Problem
}

\author{
Weizhen Rao, ${ }^{1}$ Feng Liu, ${ }^{2}$ and Shengbin Wang ${ }^{3}$ \\ ${ }^{1}$ College of Economics and Management, Shandong University of Science and Technology, Qingdao 266590, China \\ ${ }^{2}$ School of Management Science and Engineering, Dongbei University of Finance and Economics, Dalian 116025, China \\ ${ }^{3}$ Department of Marketing, Transportation and Supply Chain, School of Business and Economics, \\ North Carolina A \& T State University, Greensboro, NC 27411, USA
}

Correspondence should be addressed to Feng Liu; liufengapollo@163.com

Received 16 November 2015; Accepted 26 January 2016

Academic Editor: Sebastian Ventura

Copyright (C) 2016 Weizhen Rao et al. This is an open access article distributed under the Creative Commons Attribution License, which permits unrestricted use, distribution, and reproduction in any medium, provided the original work is properly cited.

\begin{abstract}
The classical model of vehicle routing problem (VRP) generally minimizes either the total vehicle travelling distance or the total number of dispatched vehicles. Due to the increased importance of environmental sustainability, one variant of VRPs that minimizes the total vehicle fuel consumption has gained much attention. The resulting fuel consumption VRP (FCVRP) becomes increasingly important yet difficult. We present a mixed integer programming model for the FCVRP, and fuel consumption is measured through the degree of road gradient. Complexity analysis of FCVRP is presented through analogy with the capacitated VRP. To tackle the FCVRP's computational intractability, we propose an efficient two-objective hybrid local search algorithm (TOHLS). TOHLS is based on a hybrid local search algorithm (HLS) that is also used to solve FCVRP. Based on the Golden CVRP benchmarks, 60 FCVRP instances are generated and tested. Finally, the computational results show that the proposed TOHLS significantly outperforms the HLS.
\end{abstract}

\section{Introduction}

In recent years, consequences from natural resource depletion and environmental degradation have made sustainability a more important objective than profitability for public and private transportation systems. As a result, sustainability has become one of the most popular topics studied by both practitioners and academic researchers in transportation area [1-7]. For instance, logistics service providers (LSPs) are paying increasing attention to the environmental externalities of the transportation operations [1], for example, fuel consumption and carbon dioxide equivalent $\left(\mathrm{CO}_{2} \mathrm{e}\right)$ emissions. These emissions can cause a lot of serious problems such as respiratory diseases and global warming. As pointed out in Demir et al. [5, 6], most LSPs have been using heavy- and medium-duty vehicles such as trucks and vans to perform a large amount of freight transportation operations at the local and regional levels. Trucks, whose power mainly comes from diesel engines, are one of the major sources of $\mathrm{CO}_{2} \mathrm{e}$ emissions (e.g., nitrogen oxides $\left(\mathrm{N}_{2} \mathrm{O}\right)$, particulate matter $(\mathrm{PM})$, and carbon dioxide $\left.\left(\mathrm{CO}_{2}\right)\right)$. Thus, minimizing the $\mathrm{CO}_{2}$ e emissions from trucks as well as other types of vehicles while simultaneously completing the required transportation tasks has always been a key issue for these LSPs in the past two decades. This paper aims to provide an insight and a methodology to efficiently resolve this issue.

In the most recent literature, vehicle carbon emission problems have been addressed in the area of transportation research, for example, Bigazzi and Bertini [8], Eglese and Black [9], Demir et al. [10], Dekker et al. [11], Lin et al. [12], and Demir et al. $[5,6]$. The models proposed in these papers are mainly variants of the well-known vehicle routing problem (VRP), which focuses on determining the optimal visiting routes for a fleet of vehicles in a transportation network to satisfy the transportation demands of a set of customers subject to given constraints. The classical VRP model typically minimizes either the total vehicle travelling distance/time or the total number of all vehicles dispatched in 
field [13-16]. Of all the extended VRPs, the fuel consumption VRP (FCVRP) has received most attentions over the years. The FCVRP is a variant of the capacitated VRP (CVRP); the latter imposes additional vehicle capacity constraint for a certain commodity on the regular VRP. One of the differences between the FCVRP and CVRP is that the objective function of FCVRP is to minimize total fuel consumption instead of the total traveling distance or cost. However, this difference makes FCVRP more difficult to solve using existing methodologies due to the following reasons. First, the solution space is huge as a result of additional factors (such as road gradient) considered that could affect the fuel consumption. For example, even for the same route, the fuel consumption for a vehicle traveling in one direction is different from that in the opposite direction because of the consideration of road gradient. Likewise, the complexities of basic improvement operations (2-opt, or-opt, exchange, and swap) can also increase. In this paper, we focus on the FCVRP and provide a mixed integer programming (MIP) model in which fuel consumption is measured by the degree of road gradient. We propose an efficient two-objective hybrid local search algorithm (TOHLS) to solve this problem. The TOHLS algorithm is an extension of a hybrid local search algorithm (HLS) that is also able to solve the FCVRP. In addition, we generate 60 FCVRP instances based on the Golden CVRP benchmarks. These testing instances are used to compare the performances of the TOHLS and HLS algorithms. The computational results demonstrate that the TOHLS is very effective and efficient in solving the FCVRP.

The remainder of this paper is organized as follows. In Section 2, we provide a detailed literature review of FCVRP and other related work. In Section 3, we formally formulate the problem as a MIP model and perform a complexity analysis through analogy with CVRP. After that, an efficient two-objective hybrid local search algorithm (TOHLS) is proposed to solve this model in Section 4. Section 5 performs computational studies of the proposed algorithm on 60 testing problem instances. Finally, Section 6 concludes the paper.

\section{Literature Review}

One of the most important issues in FCVRP research is how to accurately estimate the vehicle fuel consumption. There are a few studies that provide insightful estimation methodologies, for example, Bigazzi and Bertini [8], Eglese and Black [9], and Demir et al. [10]. Demir et al. [6] categorized the existing factors that can determine the fuel consumption into five classes: vehicle, environment, traffic, driver, and operations. Vehicle factors include vehicle curb weight, vehicle shape, engine size, and fuel type. Environment factors include road gradient, pavement type, and surface. Traffic factors include speed, acceleration or deceleration, and traffic congestion. Driver factors include driver aggressiveness, gear selection, and idle time. Finally, operations related factors include fleet size/mix, payload, and empty kilometers. According to Demir et al. [6], the vehicle, traffic, and environment factors are measurable and have received more research interest, whereas the driver and operations factors can hardly be measured and are sometimes treated as externalities to affect fuel consumption. In this paper, we focus on the vehicle, traffic, and environment factors. In particular, we are interested in how the vehicle type, vehicle speed, and the degree of road gradient can affect fuel consumption. First, the manufacturing information for a certain type of vehicles somehow determines their fuel consumption levels. A heavy-duty truck essentially consumes more energy than a medium-duty one assuming they are required to complete the same tasks. Second, speed is more effective than traveling distance for emission estimates since speed can affect inertia, rolling resistance, and air resistance $[6,9]$. Demir et al. [10] stated a rule of thumb for a mediumduty vehicle: if the speed is above 55 kilometers/hour, every kilometer/hour increase leads to a fuel consumption increase of 0.001 liters/kilometer approximately. An optimum driving speed at each point of a certain transportation network was obtained in Demir et al. [17] to minimize the emissions. Third, coupled with speed, road gradient is also considered to have an impact on the emissions. Intuitively, on an uphill slope, vehicles require more horsepower and fuel consumption due to gravity force than on a downhill road. According to Demier et al. [10], if a medium-duty vehicle is traveling on a 100 -kilometer road segment with $1 \%$ road slope, its fuel consumption may take up to six liters more than on the flat road. Meanwhile, in most cases fuel consumption data derived under real driving conditions are significantly different from those provided by vehicle manufacturers [18]. In sum, by combining the vehicle type, speed, and the degree of road gradient information, the fuel consumption in our model can be measured accurately. Demier et al. [6] summarized three approaches of modeling fuel consumption (with increasing complexity): factor models, macroscopic models, and microscopic models. In this research, we adopt the microscopic fuel consumption model.

FCVRP has been extensively studied in the literature. Urquhart et al. [19] focused on vehicle routing problem with time windows and studied the trade-offs between carbon dioxide savings, traveling distance, and the number of vehicles using evolutionary algorithms. The authors found out that up to $10 \%$ savings could be achieved. Bandeira et al. [20] performed three distinctive case studies in the USA and Portugal to evaluate the impact of traffic peak hours on ecofriendly routes changes. A microscopic fuel consumption model was used in their paper. The empirical results indicated that the most ecofriendly routes during off-peak and peak hours are essentially the same. In a follow-up study, Bandeira et al. [21] examined how to make the emission information available to drivers to help them select their preferred vehicle routes. It was found that the selected route has a substantial impact on pollutant emission rate and that a considerable emission reduction could be obtained from a smoother driving style. Correia et al. [22] proposed fuel consumption model to analyze ecofriendly routes in France. They utilized geographical features for eco-routing search, and the results indicated that ecoroutes were able to provide fuel savings between $1 \%$ and $36 \%$ comparing with fastest routes. Minett et al. [23] incorporated different roadway types (e.g., motorway, 
local, and provincial routes) into their fuel consumption model. Their results from the field study showed that driving in provincial routes saved for the drivers an average of 10.41 minutes and 1.25 liters of fuel comparing to driving in local routes and motorway routes, respectively.

A few papers combine the total fuel consumption and the total cost together in the objective function. Bektaş and Laporte [24] extended the VRP with time windows to a vehicle pollution routing problem by considering both total fuel consumption and total cost as the objective function. The decisions to be made in their model included the vehicle routing to serve a set of customers and the vehicle speed on each segmented route. The computational studies indicated that up to $10 \%$ energy savings could be achieved. Similarly, Demir et al. [17] also constructed a fuel consumption model and proposed an algorithm that iteratively improved the solutions to the model between vehicle routing problem with time window and a speed optimization problem. In order to precisely estimate the fuel consumption, the authors randomly selected cities from the United Kingdom and used real geographical distances to generate benchmark instances. The computational results indicated that around $10 \%$ carbon dioxide emission reduction could be achieved. Since fuel consumption and cost are inherently contradicting each other, their trade-offs must be considered when they both appear as the objectives. Demir et al. [5] studied the tradeoffs between the two factors and concluded that a fuel consumption reduction did not always come at a cost of long driving time. Ramos et al. [25] considered a multicommodity transportation network consisting of multiple depots. The authors applied a decomposition solution procedure to determine the optimal vehicle routing in order to minimize the carbon dioxide emission. The computational result of a case study indicated that up to $20 \%$ potential savings could be achieved if the company agreed to reshape the service areas and vehicle routes according to solutions. Franceschetti et al. [26] minimized the total fuel consumption, carbon dioxide emission, and driver costs under traffic congestion. They developed the optimal waiting conditions at certain locations for vehicles to avoid being stuck in traffic and to reduce the fuel consumption. Using the same instances generated in Demir et al. [17], the authors obtained a $20 \%$ reduction of total cost.

In comparison with the aforementioned studies, the main contribution of this research is a formulation of the sustainable FCVRP that incorporates road gradient into the fuel consumption model and a two-objective hybrid local search algorithm to efficiently solve the FCVRP. The complexity results are critical for connecting our problem with the numerous existing results in CVRP studies. Like most studies in VRP, the optimization techniques proposed here can also be applied to other transportation and routing problems in similar environments.

\section{Problem Formulation}

3.1. Problem Statement. The research problem we investigate is defined as follows. The transportation network consists of a distribution center (DC) denoted by 0 and a set of $n$ customers: $V_{0}=\{1,2, \ldots, n\}$. Each customer demands a given number of goods that are distributed from the DC. The DC and customers are all located in a given three-dimensional space. The position of each customer is described using the spacial coordinates with meter as unit, $\left(x_{i}, y_{i}, z_{i}\right)(0 \leq i \leq$ $n$ ), where $x_{i}$ and $y_{i}$ are horizontal coordinates and $z_{i}$ is vertical coordinate. The DC holds a set of vehicles for delivery purposes, but the exact number of vehicles to be used is unknown and must be determined optimally. Each vehicle has a loading capacity of $Q$ with kilogram $(\mathrm{kg})$ as its unit, and the curb weight of the vehicle is given by $r \cdot Q(r>$ 0 ). The demand of each customer is given by $q_{i}$ that must satisfy $0 \leq q_{i} \leq Q, 1 \leq i \leq n$. The Euclidean distance between any two customers in $V_{0}$ is calculated as $d_{i j}=$ $\sqrt{\left(x_{i}-x_{j}\right)^{2}+\left(y_{i}-y_{j}\right)^{2}+\left(z_{i}-z_{j}\right)^{2}}$. For any two customers that have the same vertical coordinates, their plane distance is hence $c_{i j}=\sqrt{\left(x_{i}-x_{j}\right)^{2}+\left(y_{i}-y_{j}\right)^{2}}$. Let $v_{i j}$ denote the speed of the vehicle traveling from customer $i$ to customer $j$ and $Q_{i j}$ denote the loading weight of the vehicle traversing the directed $\operatorname{arc}(i, j)$. In addition, we have the following assumptions.

(i) All vehicles are identical; that is, they have the same type of engine, fuel consumption rate, and loading capacity.

(ii) The road gradient between any two customers is approximated by the difference between their vertical coordinates. Each directed arc has a unique road gradient angle.

(iii) The traveling speed in the transportation network for all vehicles is the same for the same arc and different for different arcs. The details about the derivation of vehicle traveling speed are elaborated in Section 3.

(iv) The fuel consumption is determined by engine operations.

(v) Each vehicle must start and end at the DC.

(vi) The loading capacity constraint for each vehicle cannot be violated.

(vii) Each customer must be serviced once and only once and by a single vehicle.

(viii) Each vehicle has a given maximum traveling distance $L$.

We must determine an optimal number of vehicles and their optimal delivery routes such that the total fuel consumption for all vehicles is minimized. The resulting problem is called fuel consumption vehicle routing problem or FCVRP. The main difference between FCVRP and VRP model lies in the objective function where we consider minimizing total fuel consumption instead of total operations cost.

3.2. The FCVRP Model. In this subsection, we first analyze the factors that affect the fuel consumption rate (fuel consumption amount per time unit) and then formulate the problem as mixed integer programming model. 
We begin with an existing fuel consumption rate model following Barth et al. [27]:

$$
\begin{aligned}
& \mathrm{FR}=\frac{\varphi\left(k \cdot N \cdot V_{s}+P / \eta\right)}{\mu} \\
& P=\frac{P_{\text {tract }}}{\eta_{\mathrm{tf}}}+P_{\mathrm{acc}} \\
& P_{\text {tract }} \\
& =\frac{\left(M \cdot a+M \cdot g \sin \theta+0.5 C_{d} \rho S \cdot v^{2}+M \cdot g \cdot C_{r} \cos \theta\right) v}{1000},
\end{aligned}
$$

where FR is the fuel consumption rate with gallon/second as the unit while all the other denotations are the factors that determine fuel consumption. These factors can be categorized into the following two groups.

The first group contains all factors that are irrelevant to vehicle routing decisions: $\varphi$ : the air-fuel-ratio, $N$ : the operating engine speed, $\mu$ : the fuel consumption coefficient, $\eta_{\mathrm{tf}}$ : the traditional efficiency, $C_{d}$ : the aerodynamic drag coefficient, $S$ : the vehicle's frontal area size, $\rho$ : the air density, $k$ : the engine fraction factor, $V_{s}$ : the engine discharge rate, $g$ : the gravity coefficient that is equal to 9.81 in this paper, $P_{\text {acc }}$ : the other vehicle energy requirements, $\eta$ : the engine efficiency parameter, and $C_{r}$ : the roll damping coefficient. The second group contains all factors that are directly relevant to vehicle routing decisions: $d$ : the vehicle traveling distance, $M$ : the combined weight of the vehicle itself and the loaded goods, $\theta$ : the angle degree of road gradient, $v$ : the vehicle traveling speed, and $a$ : the vehicle acceleration rate that is equal to 0 in this paper.

Note that the values of $d, M, \theta$, and $v$ can change according to various distribution and routing results in reality. For example, $v$ is determined by the vehicle's route and speed limit, $M$ is determined by vehicle visiting sequences, and $d$ is determined by the degrees of road gradients in a route. Consequently, we must incorporate a dynamic driving status in the model. The vehicle driving status between customers $i$ and $j$ can be described using the second-class factors $d_{i j}, M_{i j}$, $\theta_{i j}$, and $v_{i j}$ and is shown in Figure 1.

These two groups of factors play different roles in calculating the fuel consumption coefficient $\omega_{i j}$ of the directed $\operatorname{arc}(i, j)$. Group 1 factors are constants $\left(C_{1}, C_{2}\right.$, and $C_{3}$ in the following formula), while group 2 factors are arc-specific parameters. The detailed formula for $\omega_{i j}$ is as follows:

$$
\begin{aligned}
\omega_{i j} & =\mathrm{FR} \cdot t=C_{1} \frac{d_{i j}}{v_{i j}}+C_{2}\left(M_{i j} g d_{i j} \sin \theta_{i j}+C_{3} v_{i j}^{2} d_{i j}\right. \\
& \left.+M_{i j} g d_{i j} C_{r} \cos \theta_{i j}\right),
\end{aligned}
$$

where $C_{1}=\varphi k N V_{s} / \mu, C_{2}=\varphi /\left(1000 \eta \mu \eta_{\mathrm{tf}}\right)$, and $C_{3}=$ $0.5 C_{d} \rho S$.

In formula (2), we can see that $\omega_{i j}$ is a function of $M_{i j}$, $\theta_{i j}, d_{i j}$, and $v_{i j}$. The first three values are predetermined in the

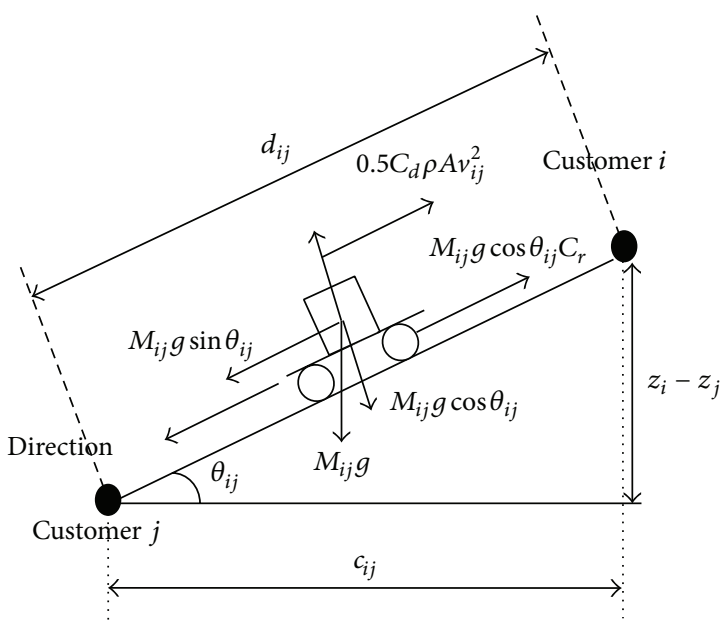

(a) Driving downhill

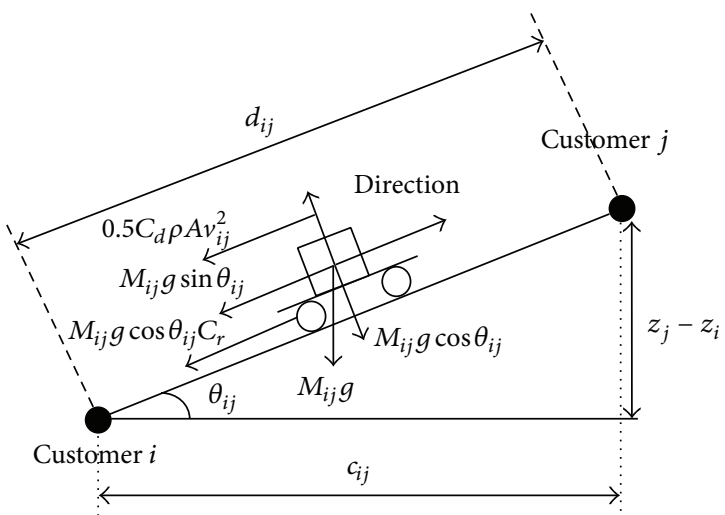

(b) Driving uphill

FIGURE 1: The vehicle driving status when going uphill and downhill.

problem, and $v_{i j}$ is determined by the two distances from each customer to the city center:

$$
v_{i j}=v_{\min }+\frac{u d_{i}+u d_{j}}{2 u d} \cdot\left(v_{\max }-v_{\min }\right),
$$

where $v_{\max }$ is the highest speed limit in the given urban area, $v_{\min }$ is the vehicle speed in the downtown area, $u d$ is the maximum distance between the border of the downtown area and the border of the suburban area, and $u d_{i}$ and $u d_{j}$ are the distances from customers $i$ and $j$ to the city center, respectively, satisfying $\max \left\{u d_{i} \mid 1 \leq i \leq n\right\} \leq u d$. City center is considered as a traffic congestion factor that affects fuel consumption. Vehicle can travel at a higher speed when it is farther away from the city center. To summarize, the vehicle fuel consumption from customer $i$ to customer $j(1 \leq i \neq j \leq$ $n$ ) can be calculated as follows:

$$
\begin{aligned}
& \omega_{i j}=f^{i j}\left(M_{i j}, d_{i j}, \theta_{i j}, v_{i j}\right)= \begin{cases}f_{1}^{i j}+f_{2}^{i j} & f_{2}^{i j}>0 \\
f_{1}^{i j} & f_{2}^{i j} \leq 0\end{cases} \\
& f_{1}^{i j}=\frac{C_{1} d_{i j}}{v_{i j}}
\end{aligned}
$$


TABLE 1: Values of parameters affecting vehicle fuel consumption.

\begin{tabular}{lcccccccccccc}
\hline Parameters & $\varphi$ & $k$ & $N$ & $V_{s}$ & $\mu$ & $g$ & $\eta_{\mathrm{tf}}$ & $C_{d}$ & $\eta$ & $S$ & $C_{r}$ & $\rho$ \\
\hline Values & 1 & 0.2 & $16-48 \mathrm{r} / \mathrm{s}$ & $2-8 \mathrm{~L}$ & 44 & 9.81 & 0.45 & 0.4 & 0.6 & $2.1-5.6 \mathrm{~m}^{2}$ & 0.01 & $1.20 \mathrm{~kg} / \mathrm{m}^{3}$ \\
\hline
\end{tabular}

$$
\begin{aligned}
f_{2}^{i j} & \\
\quad & C_{2}\left(M_{i j} g d_{i j} \sin \theta_{i j}+C_{3} v_{i j}^{2} d_{i j}+M_{i j} g d_{i j} C_{r} \cos \theta_{i j}\right) \\
M_{i j} & =r Q+Q_{i j} \\
\theta_{i j} & =\arcsin \frac{z_{j}-z_{i}}{d_{i j}},
\end{aligned}
$$

where $f_{1}^{i j}$ is the fuel consumption caused by engine usage and $f_{2}^{i j}$ is the fuel consumption caused by driving on an upward gradient road. Note that when vehicle is driving on a downward gradient road, large values of $\theta_{i j}$ can lead to negative values of $f_{2}^{i j}$. This situation makes no sense in practice, and therefore when this case occurs, we make $\omega_{i j}=$ $f_{1}^{i j}$.

The constant parameters in formula (4) can be determined from formula (2) and the empirical results in Table 1 (see Barth and Boriboonsomsin [28]).

In this paper, we consider a medium-duty vehicle, a van manufactured by Chinese Jianghuai Shuailing, Inc., and the vehicle factors are $V_{s}=4.5 \mathrm{~L}, S=7 \mathrm{~m}^{2}$, and $N=41.6 \mathrm{r} / \mathrm{s}$. Based on the values in Table 1 , we have $C_{1}=0.851, C_{2}=$ $6.313 \times 10^{-5}$, and $C_{3}=1.686$.

The relationship between vehicle velocity and fuel consumption has been widely explored in literature. One representative result can be attributed to Carslawa et al. [29]. In order to validate our model, we empirically examine the relationship between $v_{i j}$ and $\omega_{i j}$ and compare the empirical result with that obtained in Carslawa et al. [29]. We fix $C_{1}=$ $0.851, C_{2}=6.313 \times 10^{-5}, C_{3}=1.686, M_{i j}=1500 \mathrm{~kg}$, $\theta_{i j}=0$, and $d_{i j}=1000 \mathrm{~m}$ and increase $v_{i j}$ from 1.0 to $30(\mathrm{~m} / \mathrm{s})$ with 0.1 as the step length. The total number of values for $v_{i j}$ would be 291. The relationship mapping between $v_{i j}$ and $\omega_{i j}$ is drawn and compared with the mapping between vehicle speed and $\mathrm{CO}_{2}$ emission in Carslawa et al. [29]. The results are summarized in Figure 2.

From Figure 2, we can observe that our fuel consumption model has a similar curve as illustrated in Carslawa et al. [29]. As the velocity increases, the fuel consumption decreases sharply. If the velocity exceeds a certain threshold, the fuel consumption will increase, but slowly. Thus, our fuel consumption function model is verified.

We have so far derived the fuel consumption model and determined the parameters used in the model. According to formula (4), we are able to calculate the entire fuel consumption of all vehicles. Let $x_{i j k}$ be a binary variable. Consider $x_{i j k}=1$ when a directed $\operatorname{arc}(i, j)$ is serviced by vehicle $k$ and $x_{i j k}=0$ otherwise. The mixed integer programming model for the FCVRP that minimizes the total fuel consumption for all vehicles is formulated as follows:

FCVRP:

$$
\min \sum_{k=1}^{m} \sum_{i=0}^{n} \sum_{j=0}^{n} w_{i j} x_{i j k}
$$

$$
\begin{array}{ll}
\text { subject to } & \sum_{i=1}^{n} \sum_{j=1}^{n} q_{i} x_{i j k} \leq \mathrm{Q} \quad(1 \leq k \leq m) \\
& q_{j} x_{i j k} \leq Q_{i j} \leq\left(Q-q_{i}\right) x_{i j k}
\end{array}
$$

$$
(i, j) \in A(1 \leq k \leq m)
$$

$\sum_{j=0}^{n}\left(Q_{j i}-Q_{i j}\right)=q_{i} \quad i \in V_{0}$

$\sum_{i=0}^{n} \sum_{j=0}^{n} d_{i j} x_{i j k} \leq L \quad(1 \leq k \leq m)$

$$
\sum_{j=1}^{n} \sum_{k=1}^{m} x_{i j k}=1 \quad(1 \leq i \leq n)
$$

$\sum_{i=1}^{n} \sum_{k=1}^{m} x_{i j k}=1 \quad(1 \leq j \leq n)$

$\sum_{j=1}^{n} \sum_{k=1}^{m} x_{0 j k}=\sum_{i=1}^{n} \sum_{k=1}^{m} x_{i 0 k}=m \quad(1 \leq i, j \leq n)$

$$
\sum_{i, j \in H \times H} x_{i j k} \leq|H|-1, \quad H \subset V_{k}, H \neq \varnothing,
$$

$$
\begin{aligned}
& x_{i j k} \\
& = \begin{cases}1 & \text { the } k \text { th vehicle traverse } \operatorname{arc}(i, j) \\
0 & \text { otherwise }\end{cases}
\end{aligned}
$$

$$
Q_{i j} \geq 0 \quad(i, j) \in A .
$$

The objective function (5) is the total fuel consumption for a feasible delivery schedule, and $\omega_{i j}$ is calculated according to formula (4). Constraint (6) are vehicle loading capacity constraints. Constraint (7) ensure that a vehicle is not overloaded on any arc it travels. Constraint (8) indicate the loading quantity is decreased by $q_{i}$ after visiting customer $i$. Constraint (9) are the vehicle traveling distance constraints. Constraints (10) and (11) are routing constraints, meaning each customer is served by only one vehicle. Constraint (12) 


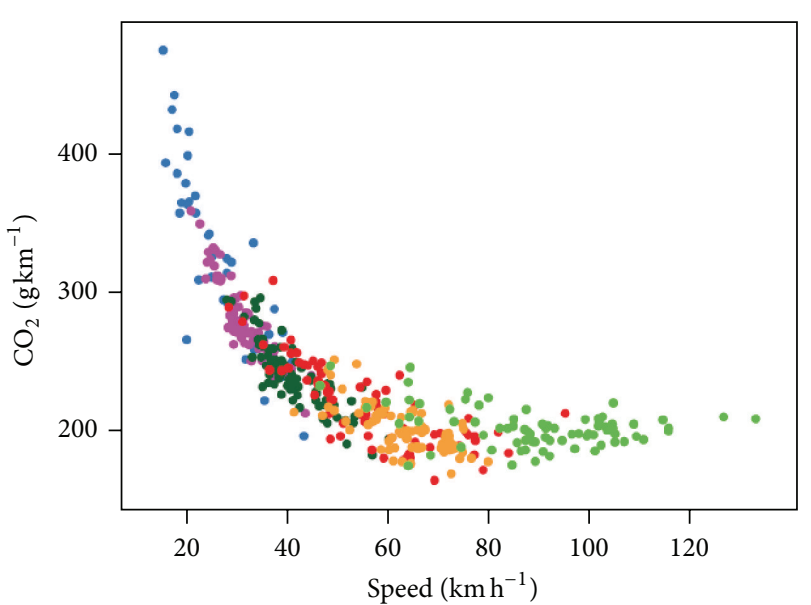

(a) $\mathrm{CO}_{2}$ emission

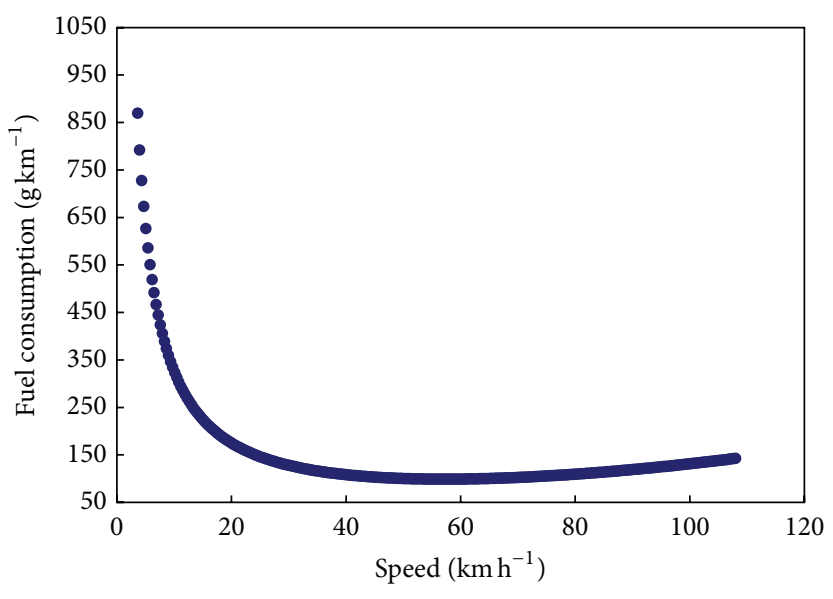

(b) Fuel consumption

FIGURE 2: The relationships between driving speed and $\mathrm{CO}_{2}$ emission and fuel consumption.

ensure all vehicles start and end at the DC. Constraint (13) ensures that each vehicle route is a simple cycle, where $V_{k}$ is the set of customers serviced by vehicle $k(1 \leq k \leq m)$. Finally constraints (14) and (15) are variable integrity and nonnegativity requirements.

3.3. Complexity Analysis of the FCVRP Model. The following theorem specifies the relationship between FCVRP and CVRP.

Theorem 1. When it holds that $r \gg 1, v_{i j}=v$ for $(i, j) \in A$, $z_{i}=z$ for $i \in V$, the FCVRP can be reduced to CVRP.

Proof. $\omega_{i j}$ in the objective function can be expressed as follows:

$$
\begin{aligned}
\omega_{i j} & =C_{1} \frac{d_{i j}}{v_{i j}}+C_{2}\left(M_{i j} g d_{i j} \sin \theta_{i j}+C_{3} v_{i j}^{2} d_{i j}\right. \\
& \left.+M_{i j} g d_{i j} C_{r} \cos \theta_{i j}\right)=C_{1} \frac{d_{i j}}{v}+C_{2}\left[C_{3} v^{2} d_{i j}\right. \\
& \left.+\left(r Q+Q_{i j}\right) g d_{i j} C_{r}\right] \approx C_{1} \frac{d_{i j}}{v}+C_{2}\left(C_{3} v^{2} d_{i j}\right. \\
& \left.+r Q g d_{i j} C_{r}\right)=\left[\frac{C_{1}}{v}+C_{2}\left(C_{3} v^{2}+r Q g C_{r}\right)\right] c_{i j} \\
& =\beta c_{i j} .
\end{aligned}
$$

Since $\beta$ is a constant that is independent of vehicle route selection, the fuel consumption for traveling from customer $i$ to customer $j$ can be replaced by the length of $\operatorname{arc}(i, j)$, $c_{i j}$. To minimize the total fuel consumption is equivalent to minimizing the total lengths of all vehicle routes, given the constraints on the vehicle traveling distances. Therefore, Theorem 1 holds.

Since CVRP is a special case of FCVRP, the computational complexity of FCVRP is higher than CVRP. The following argument elaborates the complexity analysis from two perspectives: the size of the solution space and the complexity of improvement operations in solutions.

First, let $\Omega_{E}$ and $\Omega_{C}$ denote the solution space for FCVRP and CVRP, respectively. Based on Theorem 1, we have $\Omega_{C} C$ $\Omega_{E}$. One feasible solution to CVRP is also a feasible solution to FCVRP. We use the following notations to determine the exact relationship between the two sets.

(i) $k$ is the number of vehicles.

(ii) $n_{i}^{s}$ is the number of customers serviced by vehicle $i$.

(iii) $u$ is the number of vehicles satisfying $n_{i}^{s}=1$; we have $0 \leq u \leq n$.

(iv) $k_{\min }$ is the minimum number of vehicles required when there are $u$ vehicles; each of these vehicles services only one customer in its route.

(v) $K$ is the number of those vehicles that can serve at least 2 customers in its route $\left(n_{i}^{s} \geq 2\right)$.

We have $k \in\left[k_{\min }, n\right]$, and

$$
\begin{aligned}
& k_{\min }=u+K_{\min }, \quad K_{\min }=\left\lceil\frac{\left(\sum_{i=1}^{n} q_{i}\right)-Q_{u}}{Q}\right\rceil \\
& K=k-u \geq k_{\min }-u=K_{\min } \text {, } \\
& \begin{cases}K \leq \frac{n}{2} & n \text { is even } \\
K \leq \frac{(n-1)}{2} & n \text { is odd }\end{cases} \\
& \sum_{i=1}^{k} n_{i}^{s}=\sum_{i=1}^{u} n_{i}^{s}+\sum_{i=u+1}^{k} n_{i}^{s}=u+\sum_{i=u+1}^{k} n_{i}^{s}=n \\
& \left(1 \leq s \leq U_{n}^{k}\right),
\end{aligned}
$$

where $Q_{u}$ is the total demand of $u$ customers and $U_{n}^{k}$ is the total number of ways to separate $n$ customers into $k$ groups 
that can be serviced by $k$ vehicles. $U_{n}^{k}$ is just the number of combinations $(n, k)$. For example, there are two ways to separate four customers to two groups, $2-2$ or $1-3$. It means that we can use one of the vehicles to serve two customers and the other vehicle to serve the remaining two customers or we can use one vehicle to serve one customer and the other vehicle to serve the remaining three customers.

For CVRP, we have

$$
\begin{aligned}
\left|\Omega_{C}\right| & =\sum_{k=k_{\min }}^{n}\left\{\sum_{s=1}^{U_{n}^{k}}\left[C^{s} \cdot \prod_{i=u+1}^{k} \frac{\left(n_{i}^{s}+1-1\right) !}{2}\right]\right\} \\
& =\sum_{k=k_{\min }}^{n}\left\{\sum_{s=1}^{U_{n}^{k}}\left(C^{s} \cdot \prod_{i=u+1}^{k} \frac{n_{i}^{s} !}{2}\right)\right\},
\end{aligned}
$$

where $C^{s}$ is the number of different combination ways for a certain separating method $s$.

For FCVRP, we have

$$
\begin{aligned}
\left|\Omega_{E}\right| & =\sum_{k=k_{\min }}^{n}\left\{\sum_{s=1}^{U_{n}^{k}}\left[C^{s} \cdot \prod_{i=u+1}^{k}\left(n_{i}^{s}+1-1\right) !\right]\right\} \\
& =2^{K} \sum_{k=k_{\min }}^{n}\left\{\sum_{s=1}^{U_{n}^{k}}\left(C^{s} \cdot \prod_{i=u+1}^{k} \frac{n_{i}^{s} !}{2}\right)\right\} .
\end{aligned}
$$

Thus, we have $\left|\Omega_{E}\right| \geq 2^{K_{\min }} \cdot\left|\Omega_{C}\right|$. Due to $K_{\min } \geq 1$, we are able to obtain that the number of feasible solutions to FCVRP is at least twice that to CVRP.

Next, we evaluate the four common types of solution improvement operations for FCVRP: 2-opt, or-opt, exchange, and swap. The selection criterion for these operations is whether the total fuel consumption can be improved. From Figures 3 and 4, we can see that 2-opt and or-opt operations may only change the route of one vehicle, whereas exchange and swap operations may change the routes of two vehicles.

From formula (4), $Q_{i j}$ is required to calculate the fuel consumption in FCVRP. In order to evaluate the fuel consumption increment, the changes of $Q_{i j}$ caused by the four operations must be considered. On the contrary, in CVRP only the difference between the total length of newly added arcs and that of deleted arcs needs to be considered to evaluate objective increment. Because $k_{\min } \geq 2$, we obtain the following results on the complexity for one step of operation for the four operations:

$$
\begin{aligned}
\operatorname{complexity}_{E}^{2-o p t} & =O(j+1-i-1)=O(i+p-i) \\
& =O(p) \leq O\left(n_{k}\right) \approx O\left(\frac{n}{k_{\min }}\right) \\
& \leq O(n) \\
\text { complexity }_{E}^{\text {or-opt }} & =O(t-i)=O(p+3)=O(p) \\
& \leq O\left(n_{k}\right) \approx O\left(\frac{n}{k_{\min }}\right) \leq O(n)
\end{aligned}
$$

$$
\begin{aligned}
\text { complexity }_{E}^{\text {exchange }} & =O(b)+O(d) \leq O\left(n_{k}+n_{k-1}\right) \\
& \approx O\left(\frac{2 n}{k_{\text {min }}}\right) \leq O(n) \\
\text { complexity swap }_{E} & =O(b)+O(d) \leq O\left(n_{k}+n_{k-1}\right) \\
& \approx O\left(\frac{2 n}{k_{\text {min }}}\right) \leq O(n) .
\end{aligned}
$$

According to complexity theory, these four operations complexities are $O(n)$ for FCVRP. However, the results for CVRP are merely $O(1)$. Combining the results of $\left|\Omega_{E}\right| \geq$ $2^{K_{\min }} \cdot\left|\Omega_{C}\right|\left(K_{\text {min }} \geq 1\right)$, we can conclude that FCVRP is more complicated and requires more time to solve than CVRP.

\section{A Two-Objective Hybrid Local Search Algorithm for Solving FCVRP}

In literature, capacitated vehicle routing problem is solved mainly through various heuristics. Since fuel consumption vehicle routing problem is more difficult than capacitated vehicle routing problem, these well-established heuristics for capacitated vehicle routing problem are not sufficient for our problem. In addition, it is usually required that a satisfactory solution is obtained within a reasonable computational time. Thus, in order to solve fuel consumption vehicle routing problem efficiently, we design a new algorithm that improves an existing hybrid local search approach used in both problems. This algorithm is based on the following analysis of the impact of the road gradient on the relationship between two problems.

4.1. The Impact of Road Gradient. Our proposed approach is primarily based on the analysis of the impact of the road gradient on capacitated and fuel consumption vehicle routing problems. This impact is illustrated in the following example (two scenarios) below.

A fuel consumption vehicle routing problem example consisting of one distribution center and 99 customers is generated from the benchmark instance kroa100 in TSP library. Assume that only one vehicle is required to service these 99 customers and that the location of the DC is given by coordinates $(1380,939)$. The optimal total traveling distance for kroal00 is known to be $21282 \mathrm{~m}$. The location of the city center is defined as the average coordinates of all customers and distribution center. The city radius $u d$ is the maximum distance from all the customers or the distribution center to the city center. In the first scenario, we generate all vertical coordinates $z_{i}$ for the distribution center and all customers randomly in order to add road gradient to the problem instance. These coordinates along with other parameters are summarized in Table 2.

From Table 2, the total demand of 99 customers equals $4970 \mathrm{~kg}$, and the loading capacity of each vehicle is $5000 \mathrm{~kg}$. Therefore, one vehicle is sufficient to service all customers in terms of the total loading weight. According to Theorem 1, the optimal solution to fuel consumption vehicle routing 


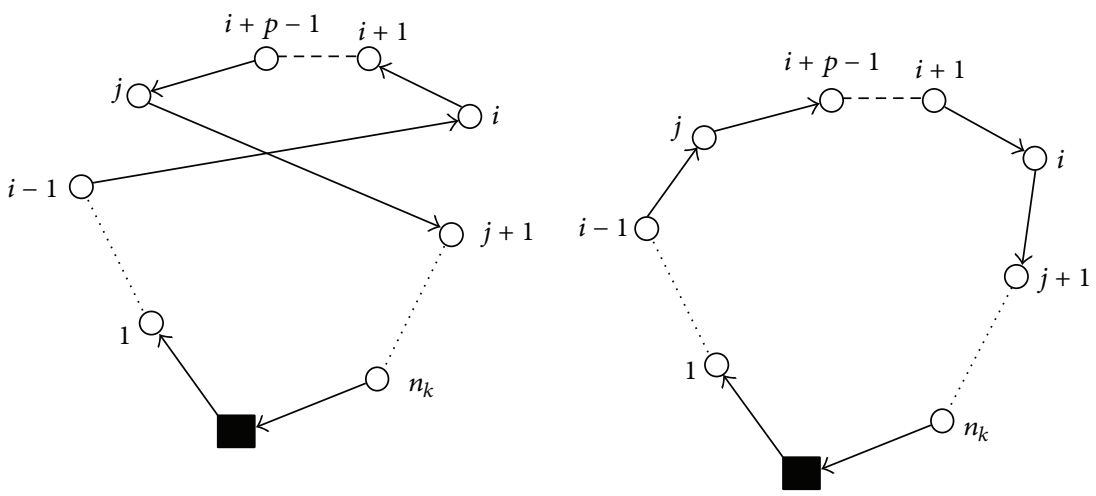

(a) 2-opt
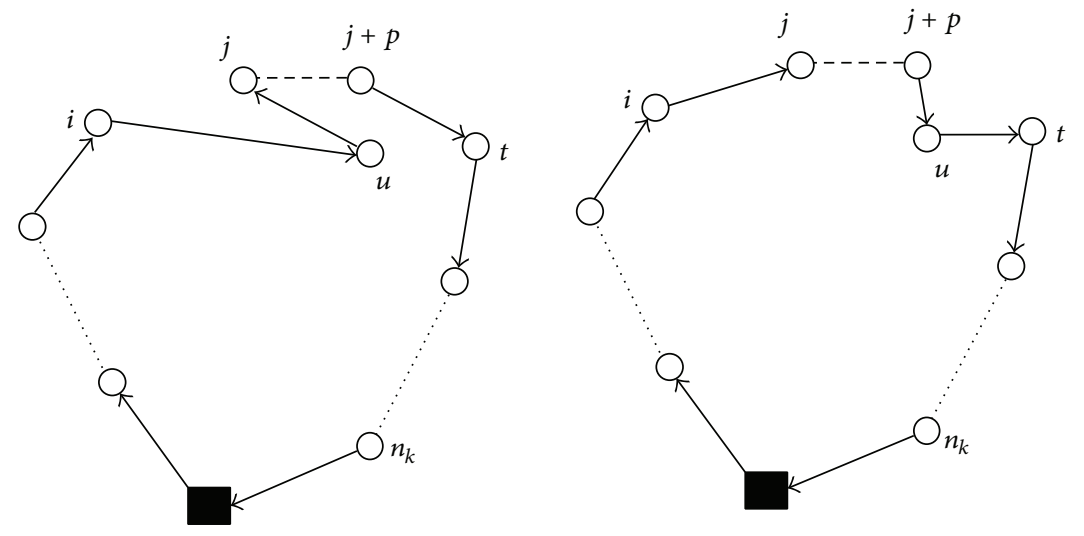

(b) or-opt

FIGURE 3: The 2-opt and or-opt operations.

TABLE 2: The problem instance generated from kroal00.

\begin{tabular}{lcccc}
\hline $\begin{array}{l}\text { DC and } \\
\text { customers }\end{array}$ & $z(\mathrm{~km})$ & $q(\mathrm{~kg})$ & $\begin{array}{c}\text { Value of } \\
\text { parameters }\end{array}$ \\
\hline 1 & 0 & 0 & $l=0.001 \mathrm{~km}$ & $C_{r}=0.01$ \\
$2-20$ & 0.02 & 30 & $r=0.5$ & $C_{1}=0.851$ \\
$21-40$ & 0.03 & 40 & $Q=5000 \mathrm{~kg}$ & $C_{2}=6.313 \times 10^{-5}$ \\
$41-60$ & 0.04 & 50 & $v_{\max }=25 \mathrm{~m} / \mathrm{s}$ & $C_{3}=1.686$ \\
$61-80$ & 0.05 & 60 & $v_{\min }=5 \mathrm{~m} / \mathrm{s}$ & \\
$81-100$ & 0.06 & 70 & $g=9.81$ & \\
\hline
\end{tabular}

problem in this case is also the optimal solution to kroa100 (capacitated vehicle routing problem). The length of the total traveling distance is $21282 \times l=21282 \times 0.0001 \mathrm{~km}=$ $21.282 \mathrm{~km}$. Associated with this optimal solution, two routing schedules with opposite directions can be generated, and their corresponding fuel consumption can also be calculated. The result is shown in Figure 5.

From Figure 5, the vehicle that travels the same distance of $21.282 \mathrm{~km}$ in opposite directions consumes different levels of fuel: $4749.513 \mathrm{~g}$ and $5054.377 \mathrm{~g}$. The difference is a result of the existence of road gradient since vehicles consume more fuel when they travel uphill than downhill. This example demonstrates that when road gradient exists, total fuel consumption of opposite vehicle routes for the same optimal sequence (thus same total distance) can have approximately $6 \%$ difference. Therefore, a simple application of existing capacitated vehicle routing problem algorithms to fuel consumption vehicle routing problem is not reliable. However, those algorithms can certainly guide solutions of fuel consumption vehicle routing problem. This is validated in the second scenario below.

In the second scenario, we further evaluate how road gradient affects the relationships between two problems. To do this, we fix all the $z_{i}$ parameter values for the distribution center and the customers and let $l$ take 101 consecutive values: $0.001,0.00101,0.00102, \ldots, 0.002(\mathrm{~km})$. These $l$ values are defined as the increment of the horizontal plane distance between two locations in the network. That is, the horizontal distance between any two customers in the entire network is increased by $l$. Through this way, we can alter the degree of road gradient by changing the ratio of vertical distance and horizontal distance. These $l$ values are used to simulate 101 geographic environments with different road gradients. In addition, based on the solutions in Figure 5, we randomly generate an integer number $n_{1}$ that satisfies $1<n_{1} \leq 91$ and then randomly rearrange the visiting sequence of 10 customers from $n_{1}$ to $n_{1}+9$ to generate 1000 neighborhood solutions. The correlation between the total traveling distance and the total fuel consumption is evaluated through the 1000 

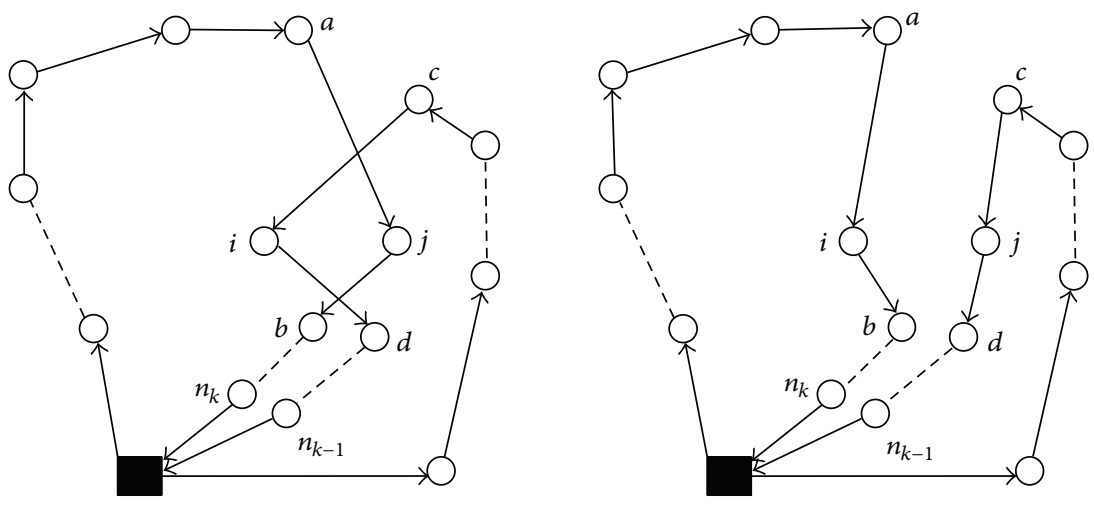

(a) Exchange
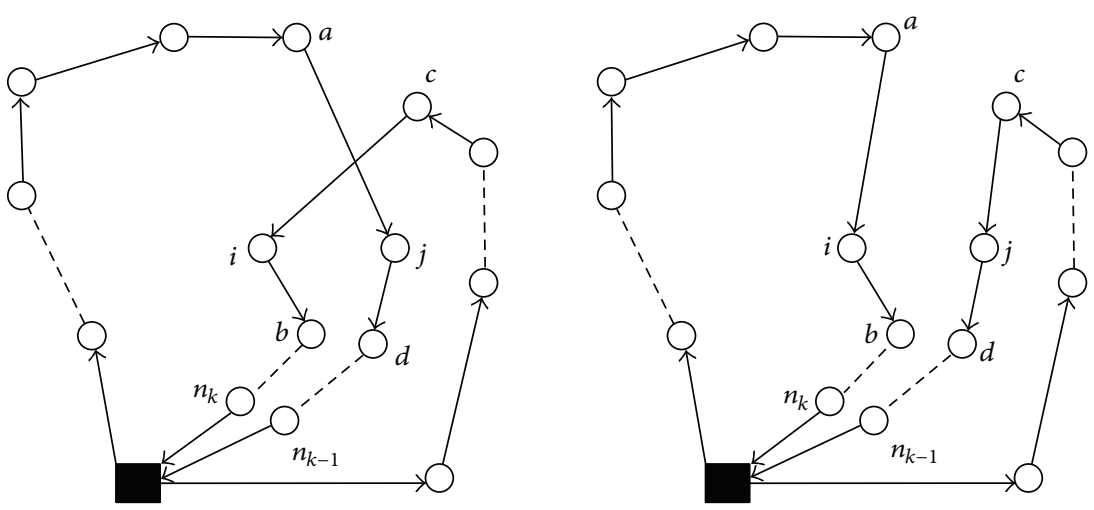

(b) Swap

FIGURE 4: The exchange and swap operations.

solutions. The correlation coefficient is defined as Coef. Besides, we also define an indicator SlopeDegree to measure the average road gradient. SlopeDegree is calculated as follows:

$$
\text { SlopeDegree }=\left\{\begin{array}{l}
\frac{\sum_{k=1}^{K} V_{k}}{n} \\
V_{k}=\sum_{i=1}^{n_{k}-1} s d_{S[i], S[i+1]}+s d_{S\left[n_{k}\right], S[1]} \\
s d_{i j}= \begin{cases}\frac{z_{i}-z_{j}}{d_{i j}} & z_{i} \geq z_{j} \\
\frac{z_{j}-z_{i}}{d_{i j}} & z_{i}<z_{j},\end{cases}
\end{array}\right.
$$

where $K$ stands for the number of vehicles in solution $S$ and $n_{k}$ stands for the number of customers served by vehicle $k$.

The mapping of SlopeDegree against Coef is shown in Figure 6. We can see that when SlopeDegree $\leq 9 \%$, Coef $>$ 0.9 and that as SlopeDegree increases, Coef decreases. When $l=0.001$ in Figure 5, SlopeDegree $=12.63 \%$ and Coef $=$ 0.81 . The details regarding the 1000 pairs of total distances and total fuel consumption are described in Figure 7.

4.2. Solving Strategy Design. In China, the requirement on the degree of road gradient for inter- and intracity highways is less than $12 \%$. In many cities, the average degree of road gradient SlopeDegree is usually less than $7 \%$. From Figure 6, when SlopeDegree $=7 \%$, the correlation coefficient between fuel consumption and traveling distance satisfies Coef $\geq 0.95$. According to the relationship, we can claim that the capacitated vehicle routing problem's optimal solutions are highly correlated with fuel consumption vehicle routing problem's optimal solutions in our case where road slope is reasonable. However, combining the first scenario, we find the difference between the two problems solutions is still significant. Thus, given a capacitated vehicle routing problem solution, we must adjust it through a way until it becomes a fuel consumption vehicle routing problem solution.

Based on the above observation, we design the twoobjective hybrid local search (TOHLS) algorithm to solve the fuel consumption vehicle routing problem. The main idea behind our approach is to divide the solution procedure into two stages, and in each stage we solve an optimization problem towards a different objective function. In the first stage, an optimal solution to capacitated vehicle routing problem, in which we minimize the total traveling distance, is obtained using a hybrid of several existing local search heuristics in literature. The obtained solution can be used in two ways to calculate the total fuel consumption. One way is to directly calculate the total fuel consumption offline using the route sequence generated from this solution. We 
Fuel consumption $5054.377 \mathrm{~g}$, distance $21.282 \mathrm{~km}$

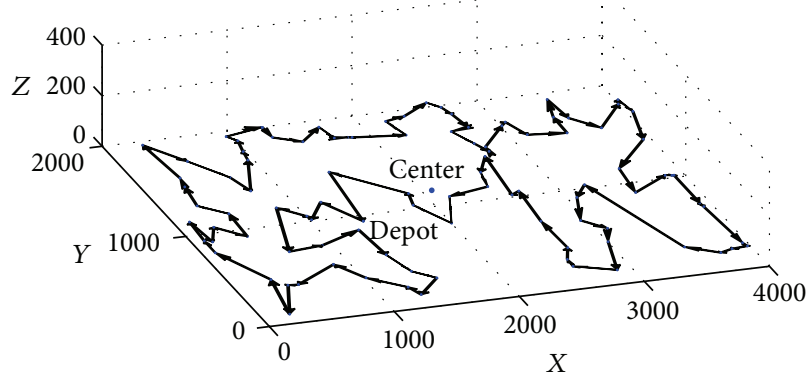

(a) Clockwise
Fuel consumption $4749.513 \mathrm{~g}$, distance $21.282 \mathrm{~km}$

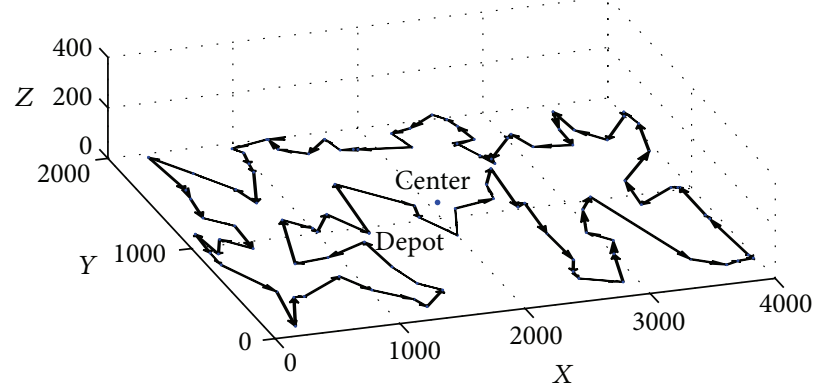

(b) Anticlockwise

FIGURE 5: The comparison of fuel consumption of driving clockwise and anticlockwise.

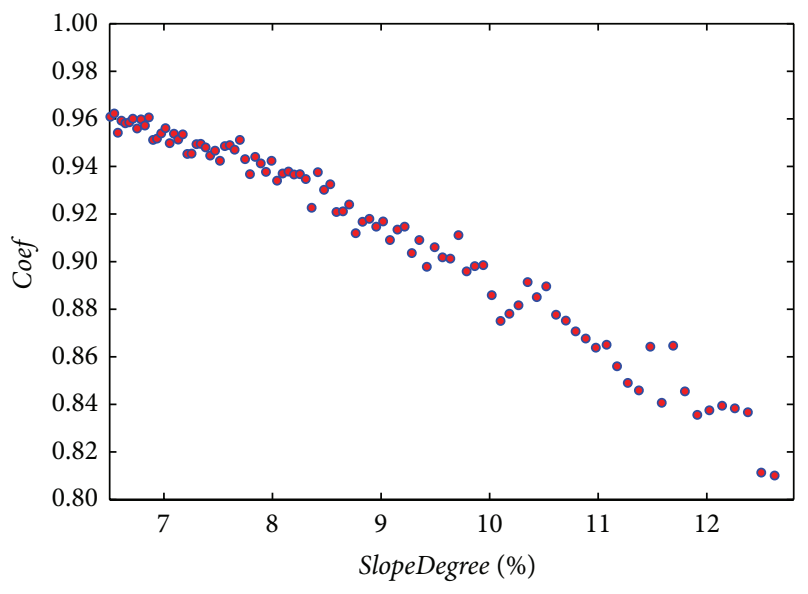

FIGURE 6: Relationship between SlopeDegree and Coef.

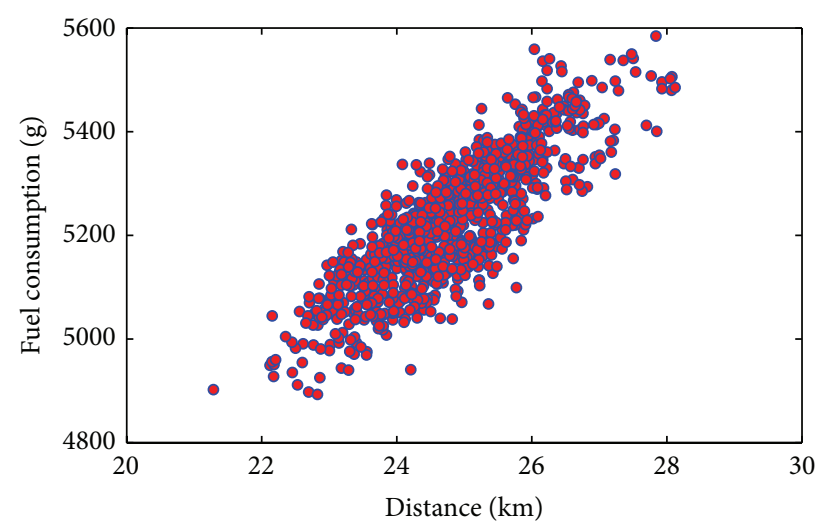

FIGURE 7: Relationship between distance and fuel consumption.

call this approach a hybrid local search (HLS) algorithm. The other way is to use it as an input for the second stage. In the second stage, the capacitated vehicle routing problem optimal solution in the first stage is improved by iteratively adjusting the traveling directions towards lower fuel consumption (new objective) until a satisfactory solution is obtained. This approach is our two-objective hybrid local search algorithm.
The details about the algorithm that incorporates 2-opt, oropt, exchange, and swap are shown in Algorithm 1.

A key issue in implementing the aforementioned approach is the allocation of limited computational resource. It is usually required in practice that the dynamic problem setting change should be completed in a given time period $T$. In order to efficiently utilize available computational resource, we allocate $T$ into the two stages based on two factors. The first factor is the ratio of the operational complexity of fuel consumption vehicle routing problem to that of capacitated vehicle routing problem, $n / k_{\min }$, and the second factor is the fuel-distance correlation coefficient, Coef. The specific allocation is as follows:

$$
\begin{aligned}
T & =T_{1}+T_{2} \\
T_{1} & =\alpha T \\
\alpha & =\frac{k_{\min } \text { Coef }}{n-\left(n-k_{\min }\right) \text { Coef }},
\end{aligned}
$$

where $T_{1}$ is the running time for Stage 1 of the two-objective hybrid local search algorithm, $T_{2}$ is the running time for Stage 2, $n$ is the number of customers, Coef is the correlation coefficient, and $k_{\min }$ is the minimum number of vehicles required in the routing schedule. The values of Coef for various SlopeDegree are shown in Table 3.

\section{Computational Studies}

5.1. Experiment Design Based on VRP Benchmark Instances. Since fuel consumption vehicle routing problem considering road gradient has rarely been studied in the VRP research community, it is difficult to directly compare our approach with the other cutting-edge algorithms by solving the same benchmark problems. Thus, we design 60 fuel consumption vehicle routing problem instances based on the 20 capacitated vehicle routing problem benchmark problems proposed in Golden et al. [30]. In the 20 problems, the number of customers ranges from 200 to 483 . All customers and the DC are located on a horizontal plane where the position of the distribution center is $(0,0)$ and all customers are within a circle that has a radius of $R$. The vertical coordinates 


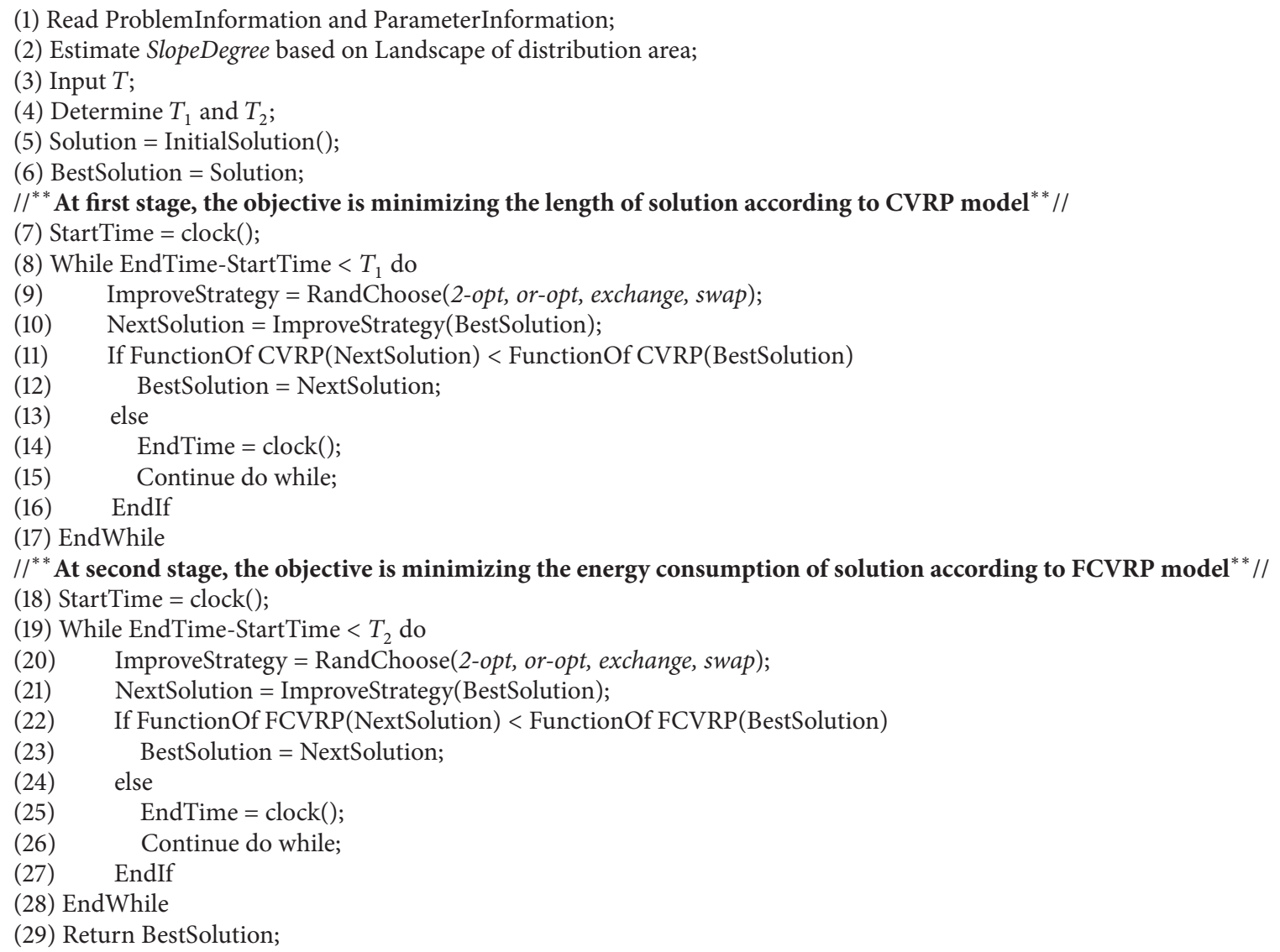

Algorithm 1: Two-objective hybrid local search algorithm.

TABLE 3: The relationship between SlopeDegree and Coef.

\begin{tabular}{lcccccccc}
\hline SlopeDegree & 0 & $(0,1 \%]$ & $(1 \%, 2 \%]$ & $(2 \%, 3 \%]$ & $(3 \%, 4 \%]$ & $(4 \%, 5 \%]$ & $(5 \%, 8 \%]$ & $\geq 8 \%$ \\
\hline Coef & 0.99 & 0.98 & 0.97 & 0.96 & 0.95 & 0.94 & 0.90 & 0.80 \\
\hline
\end{tabular}

$\left(z_{i}, 0 \leq i \leq n\right)$ and a city center are added to generate the fuel consumption vehicle routing problem instances.

(i) The DC and the city center are both located at $(0,0,0)$.

(ii) $z_{i}$ 's for the 483 customers and the DC are shown in Table 4.

(iii) The loading capacity of each vehicle $Q$ is set to be $5000 \mathrm{~kg}$, and the demand of each customer is assigned accordingly.

(iv) The arc length between any two customers is converted to three possible values, 5000, 3000 and 1000, according to the radius of location circle, $R$.

The 20 capacitated vehicle routing problem instances along with $3 R$ values produce the 60 fuel consumption vehicle routing problem instances. In order to test the performance of two-objective hybrid local search algorithm, we use a hybrid of three local search heuristics, petal, nearest neighborhood, and insertion to generate optimal capacitated vehicle routing problem solutions in the first stage. In the second stage, we locally adjust the solutions from the first stage to improve them towards the direction of reducing fuel consumption, and to finally solve the 60 instances. Recall that we can also generate fuel consumption vehicle routing problem solutions from hybrid local search by using solutions obtained from the first stage. The maximum running time limit $T$ takes 4 values: 2 minutes, 5 minutes, 10 minutes, and 30 minutes. The other parameters of fuel consumption vehicle routing problem model are shown in Tables 4 and 5. Our computational results show that two-objective hybrid local search algorithm significantly outperforms hybrid local search.

The results are shown in Tables 6-8. The problem instances are entitled as "Golden $+n+($ num $)$," where $n$ is the number of customers and num is the numth instance. In Tables 6-8, the objective function values (i.e., the total fuel consumption) obtained by hybrid local search algorithm for the four different time limits are given. However, in order to make the comparison between two-objective hybrid local search algorithm and hybrid local search more clear, we present the results of two-objective hybrid local search 
TABLE 4 : The $z_{i}$ values for DC and customers $1-483$ of 20 instances.

\begin{tabular}{|c|c|c|c|c|c|c|c|}
\hline DC and customers & $z_{i}(\mathrm{~m})$ & Customer & $z_{i}(\mathrm{~m})$ & Customer & $z_{i}(\mathrm{~m})$ & Customer & $z_{i}(\mathrm{~m})$ \\
\hline $0-25$ & 100 & $126-150$ & 75 & $251-275$ & 50 & $376-400$ & 25 \\
\hline $26-50$ & 95 & $151-175$ & 70 & $276-300$ & 45 & $401-425$ & 20 \\
\hline $51-75$ & 90 & $176-200$ & 65 & $301-325$ & 40 & $426-450$ & 15 \\
\hline 76-100 & 85 & $201-225$ & 60 & $326-350$ & 35 & $451-475$ & 10 \\
\hline $101-125$ & 80 & $226-250$ & 55 & $351-375$ & 30 & $476-483$ & 5 \\
\hline
\end{tabular}

TABLE 5: The parameter values in the model.

\begin{tabular}{lc}
\hline Parameter & Value \\
\hline$r$ & 1 \\
$Q$ & $2000 \mathrm{~kg}$ \\
$v_{\max }$ & $25 \mathrm{~m} / \mathrm{s}$ \\
$v_{\min }$ & $5 \mathrm{~m} / \mathrm{s}$ \\
$g$ & 9.81 \\
$u d$ & $R$ \\
$C_{r}$ & 0.01 \\
$C_{1}$ & 0.851 \\
$C_{2}$ & $6.313 \times 10^{-5}$ \\
$C_{3}$ & 1.686 \\
\hline
\end{tabular}

algorithm as the relative percentages of the results of hybrid local search.

5.2. Results and Analysis. From Table 6, when $z_{i}$ take values in Table 4 and $R=5000(\mathrm{~m})$, the average slope SlopeDegree of the 20 Golden instances is $0.77 \%$ which is less than $1 \%$. In this case, within given computational time two-objective hybrid local search algorithm can effectively improve the performance of hybrid local search. When $T=2,5,10$ (minutes), the results of two-objective hybrid local search algorithm on 20 instances are all better than those of hybrid local search. The average solution percentages under these three time durations are $90.13 \%, 92.92 \%$, and $95.23 \%$, respectively. As the running time increases, the effectiveness of two-objective hybrid local search algorithm unfortunately decreases. When $T=30$ (minutes), the results of two-objective hybrid local search algorithm for Golden240-2, Golden252, Golden255, Golden280, Golden399, Golden440, and Golden480-1 are slightly worse than those of hybrid local search. This is because fuel consumption vehicle routing problem is more complicated than capacitated vehicle routing problem. When the running time is sufficiently long, the performance of hybrid local search is close to that of two-objective hybrid local search algorithm. However, two-objective hybrid local search algorithm overall outperforms hybrid local search. Another observation that can be made from Table 6 is that the resulting difference between two-objective hybrid local search algorithm and hybrid local search increases as the problem size grows. This can be attributed to the fact that larger-size problems require longer running time for the algorithms to converge.

In Tables 7 and $8, R$ value is decreased to $3000(\mathrm{~m})$ and $1000(\mathrm{~m})$, while $z_{i}$ still take values in Table 4 . The average

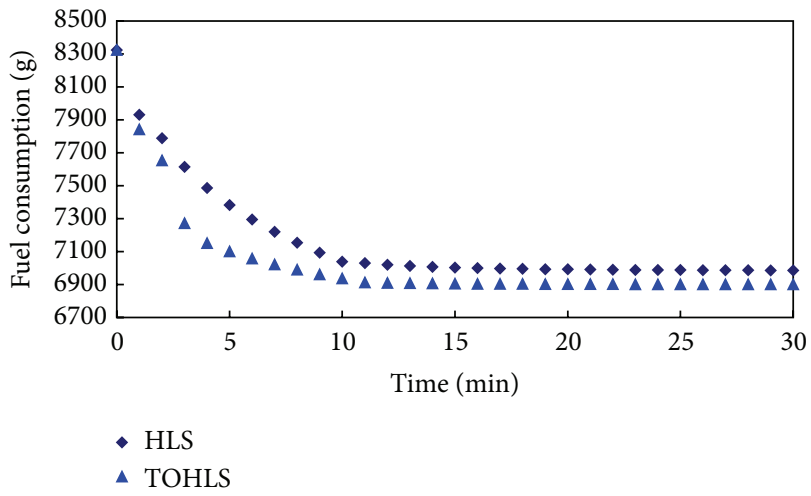

FIGURE 8: The optimization procedures of HLS and TOHLS for Golden 200 when $T=30$.

slope for the 20 problem instances is increased to $1.28 \%$ and $3.83 \%$. Although the slope is changed, the effectiveness of two-objective hybrid local search algorithm with respect to $n$ and $T$ has similar trend in Tables 7 and 8 as in Table 6. We can also see that the superiority of two-objective hybrid local search algorithm over hybrid local search is more significant as the slope increases. This seems contradictive to the previous argument that larger slope should lead to smaller correlation between fuel consumption and distance. However, this can be explained from two aspects. First, the slope limit for practical roads is less than $12 \%$, but we confine the average slope in problem instances to be less than $6 \%$. Under this level of slope, the fuel consumption is still highly correlated with routing distance (as shown in Figure 2(a)). Second, with other parameters fixed, the increase in the degree of road gradient leads to a longer converging time for the algorithm.

We select one of the testing instances, Golden200 from Table 6 with $T=30$ (minutes), and plot the solution converging processes of the two algorithms within 30 minutes in Figure 8. This figure is used to better demonstrate the superiority of two-objective hybrid local search algorithm to hybrid local search. In Figure 8, local optimal total fuel consumption obtained by hybrid local search and twoobjective hybrid local search algorithm in every minute is recorded and plotted. For this problem case, we have SlopeDegree $=0.85 \%$ from Table 6 and Coef $=0.95$ from Table 4. Based on the above information, we can obtain $T_{1}=579.66$ seconds and that $\mathrm{e}$ initial fuel consumption $=$ 8324 (g). From Figure 8, the objective function values of twoobjective hybrid local search algorithm decrease sharply in 
TABLE 6: Comparison between HLS and TOHLS when $R=5000$ (m).

\begin{tabular}{|c|c|c|c|c|c|c|c|c|c|c|}
\hline \multirow{2}{*}{ Num } & \multirow{2}{*}{ Instances } & \multirow{2}{*}{ SlopeDegree (\%) } & \multicolumn{4}{|c|}{ HLS (fuel consumption g) } & \multicolumn{4}{|c|}{ TOHLS (percentage of HLS) } \\
\hline & & & $T=2$ & $T=5$ & $T=10$ & $T=30$ & $T=2$ & $T=5$ & $T=10$ & $T=30$ \\
\hline 1 & Golden 200 & 0.85 & 15362 & 14340 & 14004 & 13764 & 95.05 & 96.30 & 96.51 & 99.20 \\
\hline 2 & Golden240-1 & 0.46 & 23257 & 21787 & 21001 & 20532 & 94.52 & 95.66 & 97.95 & 99.84 \\
\hline 3 & Golden240-2 & 0.62 & 109506 & 102754 & 99391 & 96499 & 95.01 & 96.00 & 96.15 & 101.96 \\
\hline 4 & Golden 252 & 0.62 & 112108 & 104938 & 100907 & 98835 & 94.58 & 95.71 & 95.32 & 101.21 \\
\hline 5 & Golden 255 & 0.44 & 194771 & 180280 & 174719 & 173283 & 94.32 & 96.44 & 95.94 & 100.15 \\
\hline 6 & Golden 280 & 0.55 & 20332 & 18756 & 18192 & 17650 & 93.22 & 94.40 & 96.66 & 101.68 \\
\hline 7 & Golden300 & 0.80 & 79789 & 73536 & 70472 & 69125 & 91.26 & 94.96 & 95.75 & 97.75 \\
\hline 8 & Golden320-1 & 0.61 & 25602 & 23703 & 22353 & 22237 & 90.58 & 93.99 & 95.95 & 96.14 \\
\hline 9 & Golden320-2 & 0.68 & 93881 & 87009 & 82038 & 81793 & 91.42 & 93.39 & 95.04 & 96.35 \\
\hline 10 & Golden323 & 0.56 & 152931 & 141203 & 134915 & 133108 & 90.84 & 93.42 & 94.68 & 98.60 \\
\hline 11 & Golden360-1 & 0.70 & 25209 & 22926 & 21707 & 21624 & 89.22 & 92.47 & 95.96 & 97.18 \\
\hline 12 & Golden360-2 & 1.01 & 58882 & 54023 & 51342 & 50186 & 89.37 & 91.46 & 95.85 & 96.66 \\
\hline 13 & Golden396 & 0.84 & 82670 & 75431 & 70798 & 69388 & 88.33 & 92.53 & 95.82 & 95.28 \\
\hline 14 & Golden399 & 0.84 & 125088 & 112599 & 106819 & 104470 & 88.31 & 91.73 & 95.78 & 101.08 \\
\hline 15 & Golden 400 & 0.78 & 27921 & 25116 & 23953 & 23622 & 87.98 & 91.05 & 96.15 & 96.46 \\
\hline 16 & Golden 420 & 1.25 & 49122 & 44707 & 41595 & 41042 & 85.99 & 90.30 & 94.03 & 98.54 \\
\hline 17 & Golden 440 & 0.86 & 29214 & 26152 & 24487 & 24001 & 85.97 & 90.74 & 93.02 & 101.06 \\
\hline 18 & Golden480-1 & 0.95 & 27753 & 24649 & 23155 & 22552 & 84.85 & 89.12 & 94.48 & 100.61 \\
\hline 19 & Golden $480-2$ & 1.03 & 73902 & 66191 & 61692 & 60096 & 85.61 & 89.63 & 92.02 & 97.52 \\
\hline \multirow[t]{2}{*}{20} & Golden 483 & 0.85 & 103757 & 93077 & 85876 & 84082 & 86.27 & 89.15 & 91.53 & 95.74 \\
\hline & Average & 0.77 & - & - & - & - & 90.14 & 92.92 & 95.23 & 98.65 \\
\hline
\end{tabular}

TABLE 7: Comparison between HLS and TOHLS when $R=3000(\mathrm{~m})$.

\begin{tabular}{|c|c|c|c|c|c|c|c|c|c|c|}
\hline \multirow{2}{*}{ Num } & \multirow{2}{*}{ Instances } & \multirow{2}{*}{ SlopeDegree (\%) } & \multicolumn{4}{|c|}{ HLS (fuel consumption g) } & \multicolumn{4}{|c|}{ TOHLS (percentage of HLS) } \\
\hline & & & $T=2$ & $T=5$ & $T=10$ & $T=30$ & $T=2$ & $T=5$ & $T=10$ & $T=30$ \\
\hline 1 & Golden 200 & 1.42 & 9983 & 9339 & 9129 & 9029 & 94.32 & 95.79 & 96.88 & 99.11 \\
\hline 2 & Golden240-1 & 0.77 & 15435 & 14540 & 13899 & 13810 & 93.74 & 94.86 & 95.48 & 99.29 \\
\hline 3 & Golden240-2 & 1.03 & 67896 & 63440 & 61547 & 60481 & 92.61 & 95.48 & 95.52 & 96.24 \\
\hline 4 & Golden 252 & 1.03 & 69959 & 64928 & 62091 & 62468 & 92.22 & 94.34 & 94.86 & 95.58 \\
\hline 5 & Golden 255 & 0.74 & 118322 & 109121 & 106592 & 105149 & 93.11 & 95.40 & 95.90 & 98.63 \\
\hline 6 & Golden 280 & 0.91 & 13350 & 12445 & 11908 & 11803 & 91.82 & 94.07 & 94.31 & 97.20 \\
\hline 7 & Golden 300 & 1.34 & 50545 & 46835 & 44908 & 44338 & 90.39 & 93.66 & 94.41 & 96.02 \\
\hline 8 & Golden320-1 & 1.02 & 17023 & 15794 & 14930 & 14553 & 90.48 & 92.43 & 94.86 & 100.15 \\
\hline 9 & Golden320-2 & 1.13 & 59402 & 54070 & 52388 & 51780 & 90.08 & 92.75 & 96.41 & 95.18 \\
\hline 10 & Golden 323 & 0.93 & 93293 & 85550 & 81135 & 80035 & 89.97 & 92.84 & 94.43 & 95.61 \\
\hline 11 & Golden360-1 & 1.17 & 16717 & 15115 & 14527 & 14134 & 88.93 & 91.87 & 94.11 & 95.32 \\
\hline 12 & Golden360-2 & 1.69 & 39053 & 35573 & 33553 & 33521 & 86.99 & 91.22 & 93.09 & 98.01 \\
\hline 13 & Golden396 & 1.41 & 53097 & 47672 & 45872 & 44972 & 87.73 & 91.10 & 91.23 & 98.94 \\
\hline 14 & Golden399 & 1.41 & 76742 & 70105 & 65006 & 63782 & 86.67 & 89.76 & 92.27 & 96.44 \\
\hline 15 & Golden 400 & 1.30 & 18574 & 16678 & 15825 & 15412 & 87.68 & 90.96 & 93.04 & 94.67 \\
\hline 16 & Golden 420 & 2.08 & 34960 & 31362 & 29610 & 29080 & 84.86 & 89.15 & 91.19 & 93.99 \\
\hline 17 & Golden 440 & 1.43 & 19582 & 17665 & 16584 & 16197 & 85.65 & 90.22 & 91.69 & 98.27 \\
\hline 18 & Golden480-1 & 1.59 & 18661 & 16569 & 15587 & 15049 & 83.66 & 87.86 & 92.20 & 95.61 \\
\hline 19 & Golden480-2 & 1.72 & 48506 & 43162 & 40209 & 39808 & 83.34 & 87.72 & 90.92 & 95.39 \\
\hline \multirow[t]{2}{*}{20} & Golden 483 & 1.41 & 63871 & 56936 & 53273 & 52020 & 83.88 & 88.47 & 92.92 & 96.05 \\
\hline & Average & 1.28 & - & - & - & - & 88.91 & 92.00 & 93.79 & 96.79 \\
\hline
\end{tabular}


TABLE 8: Comparison between HLS and TOHLS when $R=1000$ (m).

\begin{tabular}{|c|c|c|c|c|c|c|c|c|c|c|}
\hline \multirow{2}{*}{ Num } & \multirow{2}{*}{ Instances } & \multirow{2}{*}{ SlopeDegree (\%) } & \multicolumn{4}{|c|}{ HLS (fuel consumption g) } & \multicolumn{4}{|c|}{ TOHLS (percentage of HLS) } \\
\hline & & & $T=2$ & $T=5$ & $T=10$ & $T=30$ & $T=2$ & $T=5$ & $T=10$ & $T=30$ \\
\hline 1 & Golden 200 & 4.25 & 5031 & 4755 & 4540 & 4527 & 92.11 & 93.04 & 94.31 & 98.37 \\
\hline 2 & Golden240-1 & 2.30 & 7914 & 7443 & 7070 & 6980 & 92.54 & 95.00 & 95.05 & 94.72 \\
\hline 3 & Golden240-2 & 3.10 & 26936 & 25023 & 24356 & 23936 & 91.07 & 92.99 & 93.08 & 98.58 \\
\hline 4 & Golden 252 & 3.08 & 28830 & 27057 & 25759 & 25705 & 91.39 & 93.54 & 93.24 & 99.22 \\
\hline 5 & Golden 255 & 2.21 & 42113 & 39456 & 37685 & 37518 & 92.96 & 94.40 & 96.44 & 95.84 \\
\hline 6 & Golden 280 & 2.74 & 7045 & 6508 & 6231 & 6202 & 91.74 & 93.70 & 96.71 & 95.79 \\
\hline 7 & Golden300 & 4.01 & 23740 & 22107 & 20943 & 20607 & 88.71 & 90.40 & 92.69 & 96.77 \\
\hline 8 & Golden320-1 & 3.06 & 8941 & 8138 & 7851 & 7771 & 89.85 & 92.41 & 93.49 & 92.93 \\
\hline 9 & Golden320-2 & 3.38 & 26673 & 24542 & 23180 & 23232 & 90.12 & 91.07 & 93.53 & 96.97 \\
\hline 10 & Golden 323 & 2.80 & 33992 & 31158 & 29856 & 29131 & 90.31 & 91.61 & 95.54 & 93.69 \\
\hline 11 & Golden360-1 & 3.52 & 8805 & 8082 & 7652 & 7480 & 87.53 & 89.98 & 92.43 & 92.40 \\
\hline 12 & Golden360-2 & 5.07 & 22894 & 21090 & 19853 & 19529 & 86.09 & 88.43 & 89.98 & 94.14 \\
\hline 13 & Golden396 & 4.22 & 26017 & 23479 & 22470 & 21643 & 85.28 & 88.65 & 91.27 & 93.27 \\
\hline 14 & Golden399 & 4.22 & 28684 & 25974 & 24655 & 24092 & 86.14 & 87.81 & 92.28 & 93.48 \\
\hline 15 & Golden 400 & 3.91 & 9716 & 8805 & 8250 & 8177 & 85.54 & 88.54 & 90.49 & 96.86 \\
\hline 16 & Golden 420 & 6.24 & 22858 & 20569 & 19385 & 19022 & 81.61 & 85.18 & 89.50 & 90.21 \\
\hline 17 & Golden440 & 4.30 & 10397 & 9319 & 8831 & 8539 & 85.32 & 86.89 & 90.22 & 92.46 \\
\hline 18 & Golden $480-1$ & 4.75 & 10139 & 9093 & 8375 & 8296 & 83.44 & 86.36 & 91.15 & 91.12 \\
\hline 19 & Golden480-2 & 5.15 & 25464 & 22499 & 21190 & 20716 & 82.06 & 86.61 & 91.06 & 91.72 \\
\hline \multirow[t]{2}{*}{20} & Golden483 & 4.23 & 24684 & 22102 & 20643 & 20038 & 84.01 & 86.84 & 91.92 & 92.35 \\
\hline & Average & 3.83 & - & - & - & - & 87.89 & 90.17 & 92.72 & 94.54 \\
\hline
\end{tabular}

the first stage, because the correlation coefficient is 0.95 (very significant) and minimizing the total traveling distance can minimize the total fuel consumption.

\section{Conclusions}

In this paper, we study the FCVRP in which road gradient is considered as one of the factors to measure fuel consumption. After constructing an MIP model, we present complexity analysis for this FCVRP based on comparison with CVRP. To solve this model, we propose an efficient algorithm called TOHLS. This algorithm is based on a hybrid local search algorithm (HLS) that is also used in this paper to solve FCVRP. Based on the existing CVRP testing problems, 60 FCVRP instances are generated and solved by both HLS and TOHLS. The computational results show that the proposed TOHLS has a better performance than the HLS algorithm in terms of solution qualities.

Some important concluding remarks can be summarized as follows. First, the size of solution space of FCVRP is $2^{K_{\min }}$ times that of CVRP, and the complexities of common improvement operations including 2-opt, or-opt, exchange, and swap are $n$ times that in CVRP. Second, TOHLS algorithm can effectively improve the performance of HLS and reduce the total fuel consumption by $2 \%-13 \%$. The improvement is more significant for problem instances with limited response time, large number of customers, and average road gradient. Third, it is indicated that the TOHLS is particularly appropriate for FCVRP problems that have large sizes, consider road gradients, and require quick response. Fourthly, it is also worth pointing out that the idea behind the proposed TOHLS can be applied to improve algorithms other than HLS. Thus, one of the most promising future research directions is to apply the two-objective idea to various existing heuristics in CVRP to improve their algorithm performances.

There are also several limitations in our research. One of our assumptions is that the road gradient between any two customers is approximated by the difference between their vertical coordinates. This assumption may not be appropriate in the case where there are two city centers located at same geographic attitude but with a mountain somewhere in the middle. In this scenario, fuel consumption for vehicles traveling between the two city centers should be more than the one we proposed. Therefore, experimentally determining the more accurate fuel consumption in such case shall be one of the future research directions. In addition, our current modeling approach simplifies the speed factors and patterns by assuming that speeds are determined by the distance to the city center. However, in practice there are often more than one-road sections connecting customers with the city center, and these road sections often have different characteristics and traffic performance levels. In such case, it will be much more complicated to model the exact speeds between different locations. Thus, a thorough investigation on the speed patterns from a more practical point of view is needed 
for the future studies. Last but not least, in order to implement our approach, we can utilize the current available real-time traffic data sources, such as FCD, loop detectors, and GPS, to facilitate parameterizing our model. In particular, given the location and transportation situation of a city center, instead of simulation, it could easily adapt our model by generating real traffic data from these resources to be used for calculating the gradient, the speed, the fuel consumption, and the other related parameters. These data can be further used as inputs in our solution approach. And, finally, the optimal fuel consumption could be achieved by selecting the appropriate vehicle routes under the guidance of these advanced devices.

\section{Conflict of Interests}

The authors declare that there is no conflict of interests regarding the publication of this paper.

\section{Acknowledgments}

The authors thank the editor and two anonymous reviewers for their constructive comments, which helped them greatly improve the paper. They also gratefully acknowledge funding provided by the Promotion Research Fund for Excellent Young and Middle-Aged Scientists of Shandong Province (2014BSB01142), National Natural Science Foundation of China (71502026), and the Ministry of Education of Humanities and Social Science Project (15YJC630103).

\section{References}

[1] C. Schreyer, C. Schneider, M. Maibach, W. Rothengatter, C. Doll, and D. Schmedding, "External costs of transport: update study," Tech. Rep., INFRAS, 2004.

[2] M. Figliozzi, "Emissions minimization vehicle routing problem," in Proceedings of the 89th Transportation Research Board Annual Meeting, Washington, DC, USA, January 2010.

[3] D. Gulczynski, B. Golden, and E. Wasil, "The period vehicle routing problem: new heuristics and real-world variants," Transportation Research E: Logistics and Transportation Review, vol. 47, no. 5, pp. 648-668, 2011.

[4] E. Wygonik and A. Goodchild, "Using a GIS-based emissions minimization vehicle routing problem with time windows (EVRPTW) model to evaluate $\mathrm{CO}_{2}$ emissions and cost trade offs in a case study of a an urban delivery system," in Proceedings of the 90th Transportation Resarch Board Annual Meeting, Washington, DC, USA, January 2011.

[5] E. Demir, T. Bektaş, and G. Laporte, “The bi-objective pollutionrouting problem," European Journal of Operational Research, vol. 232, no. 3, pp. 464-478, 2014.

[6] E. Demir, T. Bektaş, and G. Laporte, "A review of recent research on green road freight transportation," European Journal of Operational Research, vol. 237, no. 3, pp. 775-793, 2014.

[7] Y. Huang, K. Wang, T. Zhang, and C. Pang, "Green supply chain coordination with greenhouse gases emissions management: a game-theoretic approach," Journal of Cleaner Production, vol. 112, Part 3, pp. 2004-2014, 2016.
[8] A. Y. Bigazzi and R. L. Bertini, "Adding green performance metrics to a transportation data archive," Transportation Research Record, vol. 2121, no. 1, pp. 30-40, 2009.

[9] R. W. Eglese and D. Black, "Optimizing the routing of vehicles," in Green Logistics: Improving the Environmental Sustainability of Logistics, A. McKinnon, S. Cullinane, M. Browne, and W. Whiteing, Eds., pp. 215-228, Kogan Page, London, UK, 2010.

[10] E. Demir, T. Bektaş, and G. Laporte, "A comparative analysis of several vehicle emission models for road freight transportation," Transportation Research Part D: Transport and Environment, vol. 16, no. 5, pp. 347-357, 2011.

[11] R. Dekker, J. Bloemhof, and I. Mallidis, "Operations Research for green logistics-an overview of aspects, issues, contributions and challenges," European Journal of Operational Research, vol. 219, no. 3, pp. 671-679, 2012.

[12] C. Lin, K. L. Choy, G. T. S. Ho, S. H. Chung, and H. Y. Lam, "Survey of green vehicle routing problem: past and future trends," Expert Systems with Applications, vol. 41, no. 4, pp. 11181138, 2013.

[13] K. C. Tan, L. H. Lee, and K. Ou, "Hybrid genetic algorithms in solving vehicle routing problems with time window constraints," Asia-Pacific Journal of Operational Research, vol. 18, no. 1, pp. 121-130, 2001.

[14] J. Zhang, J. Tang, and R. Y. K. Fung, "A scatter search for multidepot vehicle routing problem with weight-related cost," AsiaPacific Journal of Operational Research, vol. 28, no. 3, pp. 323348, 2011.

[15] B. L. Hollis and P. J. Green, "Real-life vehicle routing with time windows for visual attractiveness and operational robustness," Asia-Pacific Journal of Operational Research, vol. 29, no. 4, Article ID 1250017, 2012.

[16] K. Wang, C. Ye, and A. Ning, "Achieving better solutions for vehicle routing problem involving split deliveries and pickups using a competitive decision algorithm," Asia-Pacific Journal of Operational Research, vol. 32, Article ID 1550022, 2015.

[17] E. Demir, T. Bektaş, and G. Laporte, "An adaptive large neighborhood search heuristic for the Pollution-Routing Problem," European Journal of Operational Research, vol. 223, no. 2, pp. 346-359, 2012.

[18] L. Pelkmans and P. Debal, "Comparison of on-road emissions with emissions measured on chassis dynamometer test cycles," Transportation Research Part D: Transport and Environment, vol. 11, no. 4, pp. 233-241, 2006.

[19] N. Urquhart, E. Hart, and C. Scott, "Building low $\mathrm{CO}_{2}$ solutions to the vehicle routing problem with time windows using an evolutionary algorithm," in Proceedings of the 6th IEEE Congress on Evolutionary Computation (CEC '10), pp. 1-6, Barcelona, Spain, July 2010.

[20] J. Bandeira, D. O. Carvalho, A. J. Khattak, N. M. Rouphail, and M. C. Coelho, "A comparative empirical analysis of ecofriendly routes during peak and off-peak hours," in Proceedings of the Transportation Research Board 91st Annual Meeting, Washington, DC, USA, 2012.

[21] J. Bandeira, T. G. Almeida, A. J. Khattak, N. M. Rouphail, and M. C. Coelho, "Generating emissions information for route selection: experimental monitoring and routes characterization," Journal of Intelligent Transportation Systems, vol. 17, no. 1, pp. 3-17, 2013.

[22] A. Correia, S. Amaya, S. Meyer, M. Kumagai, and M. Okude, "Eco routing for European market," in Proceedings of the 17th World Congress and Exhibition on Intelligent Transport Systems and Services (ITS '10), Busan, South Korea, 2010. 
[23] C. F. Minett, A. M. Salomons, W. Daamen, B. Van Arem, and S. Kuijpers, "Eco-routing: comparing the fuel consumption of different routes between an origin and destination using field test speed profiles and synthetic speed profiles," in Proceedings of the IEEE Forum on Integrated and Sustainable Transportation Systems (FISTS '11), pp. 32-39, Vienna, Austria, July 2011.

[24] T. Bektaş and G. Laporte, "The pollution-routing problem," Transportation Research Part B: Methodological, vol. 45, no. 8, pp. 1232-1250, 2011.

[25] T. R. P. Ramos, M. I. Gomes, and A. P. Barbosa-Povoa, "Minimizing $\mathrm{CO}_{2}$ emissions in a recyclable waste collection system with multiple depots," in Proceedings of the EUROMA/POMS Joint Conference, Amsterdam, The Netherlands, 2012.

[26] A. Franceschetti, D. Honhon, T. Van Woensel, T. Bektaş, and G. Laporte, "The time-dependent pollution-routing problem," Transportation Research Part B: Methodological, vol. 56, pp. 265-293, 2013.

[27] M. Barth, T. Younglove, and G. Scora, "Development of a heavyduty diesel modal emissions and fuel consumption model," Tech. Rep., California Partners for Advanced Transit and Highways, San Francisco, Calif, USA, 2005.

[28] M. Barth and K. Boriboonsomsin, "Real-world $\mathrm{CO}_{2}$ impacts of traffic congestion," Transportation Research Record, vol. 2058, no. 11, pp. 163-171, 2008.

[29] D. C. Carslaw, P. S. Goodman, F. C. H. Lai, and O. M. J. Carsten, "Comprehensive analysis of the carbon impacts of vehicle intelligent speed control," Atmospheric Environment, vol. 44, no. 23, pp. 2674-2680, 2010.

[30] B. Golden, J. Wasil, and I. M. Kelly, Fleet Management and Logistics, Kluwer Academic Publishers, Boston, Mass, USA, 1998. 

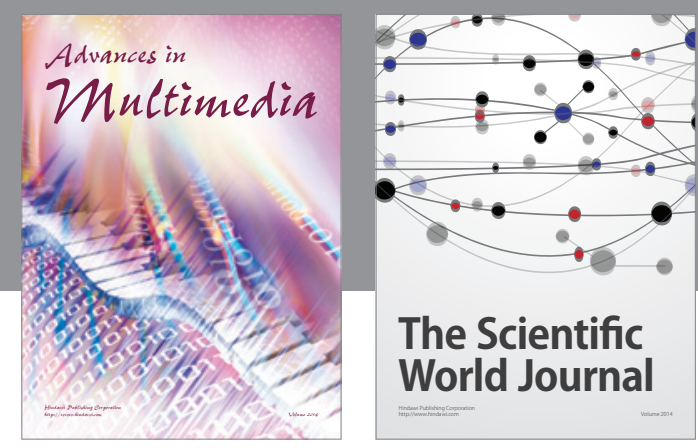

The Scientific World Journal
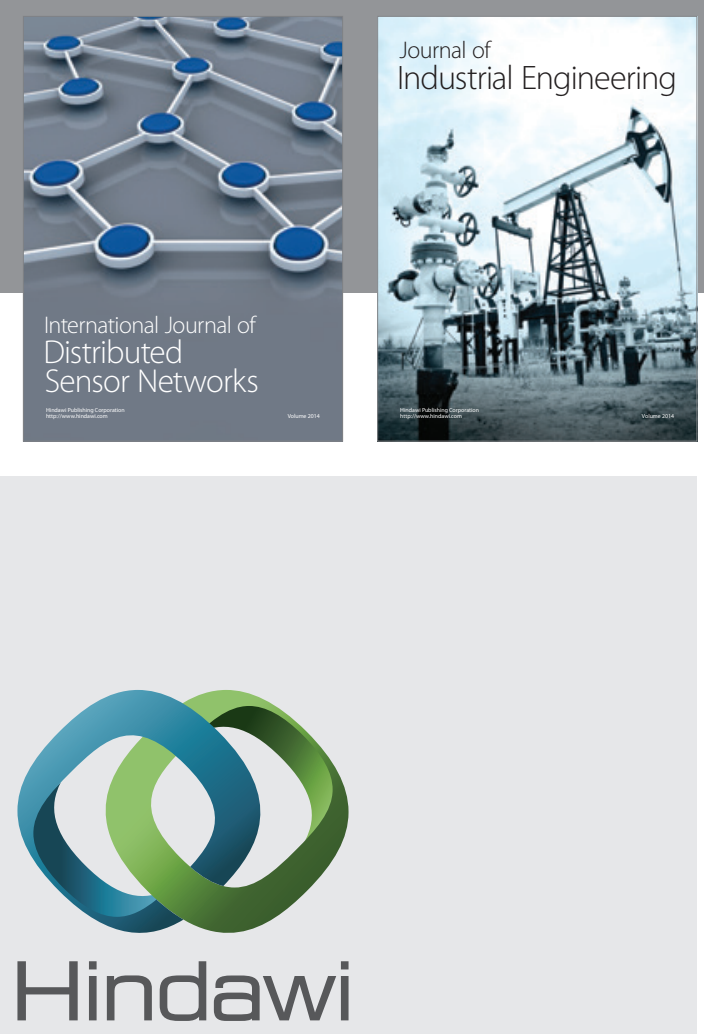

Submit your manuscripts at

http://www.hindawi.com

\section{Computer Networks} and Communications
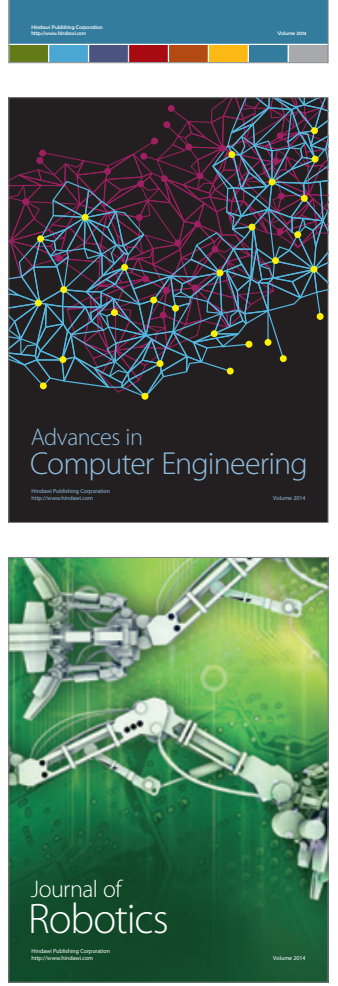
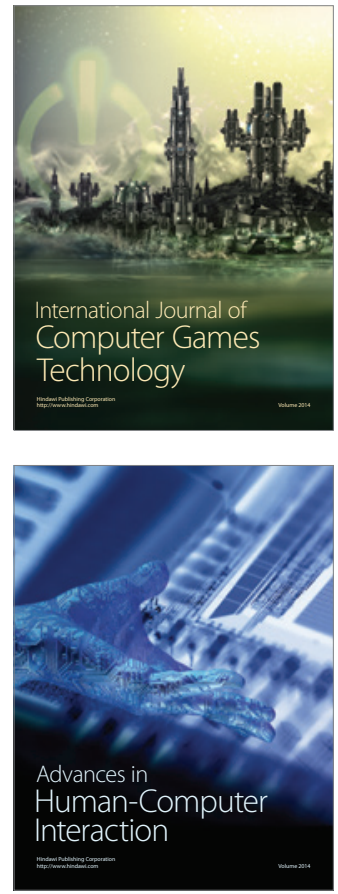
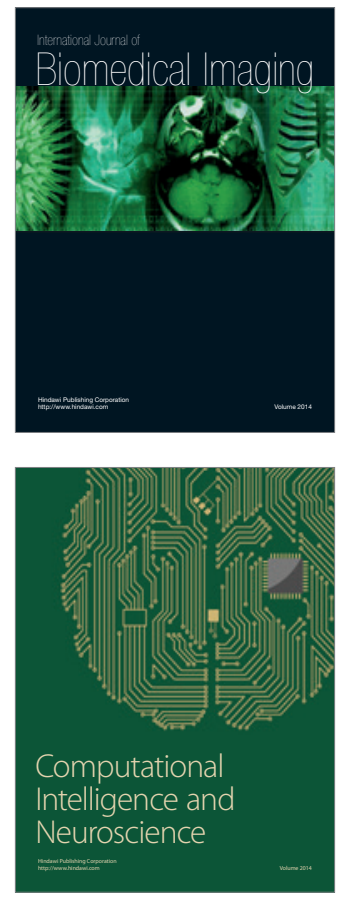
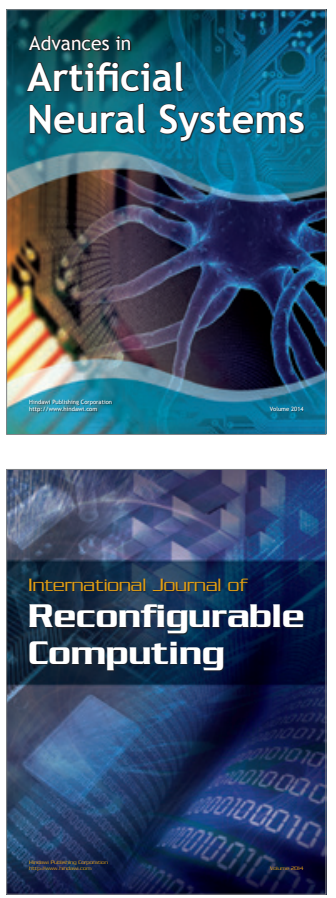
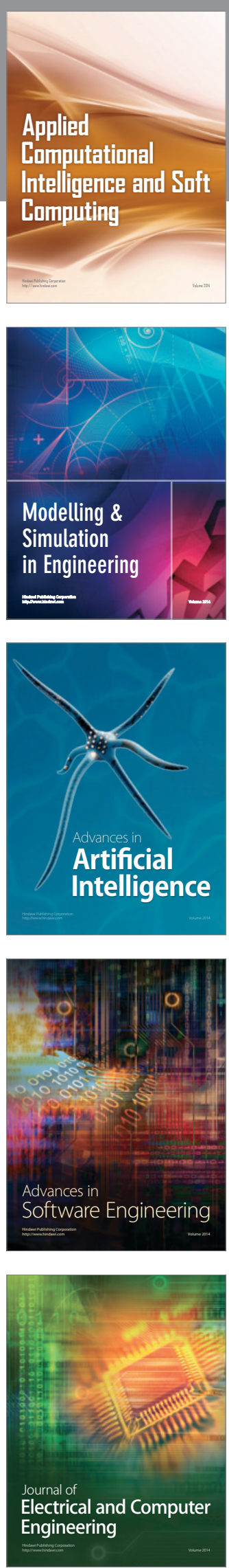